\title{
A non-homogeneous constitutive model for human blood. Part I: model derivation and steady flow
}

\begin{tabular}{|r|l|}
\hline Journal: & Journal of Fluid Mechanics \\
\hline Manuscript ID: & JFM-07-S-0327.R2 \\
\hline mss type: & Standard \\
\hline Complete List of Authors: & $\begin{array}{l}\text { Owens, Robert; Université de Montréal, Mathématiques et } \\
\text { statistique } \\
\text { Moyers-Gonzalez, Miguel; University of Durham, Department of } \\
\text { Mathematical Sciences } \\
\text { Fang, Jiannong; EPFL }\end{array}$ \\
\hline Keyword: & $\begin{array}{l}\text { Blood flow < Biological Fluid Dynamics, Capsule/cell dynamics < } \\
\text { Biological Fluid Dynamics, Rheology < Non-Newtonian Flows }\end{array}$ \\
\hline &
\end{tabular}

\section{5) ScholarONE" \\ Manuscript Central}




\title{
A non-homogeneous constitutive model for human
}

\author{
blood. Part I: model derivation and steady flow. \\ MIGUEL MOYERS-G ONZALEZ ${ }^{1} \dagger, \quad$ ROBERT G. OWENS ${ }^{1} \ddagger$ \\ AND JIA N N O N G F A N G \\ ${ }^{1}$ Département de mathématiques et de statistique, Université de Montréal, CP 6128 succ. Centre-Ville, \\ Montréal QC H3C 3J7, Canada \\ ${ }^{2}$ GEOLEP-ICARE-ENAC, Ecole Polytechnique Fédérale de Lausanne, 1015 Lausanne, Switzerland
}

(Received 12 August 2008)

The earlier constitutive model of Fang \& Owens (2006) and Owens (2006) is extended in scope to include non-homogeneous flows of healthy human blood. Application is made to steady axisymmetric flow in rigid walled tubes. The new model features stress-induced cell migration in narrow tubes and accurately predicts the Fåhraeus-Lindqvist effect (Fåhraeus \& Lindqvist (1931)) whereby the apparent viscosity of healthy blood decreases as a function of tube diameter in sufficiently small vessels. That this is due to the development of a slippage layer of cell-depleted fluid near the vessel walls and a decrease in the tube hematocrit (Fåhraeus (1929)) is demonstrated from the numerical results. Although clearly influential, the reduction in tube hematocrit observed in small vessel blood flow (the so-called Fåhraeus effect) does not therefore entirely explain the Fåhraeus-Lindqvist effect.

Keywords: Non-homogeneous flows, Fåhraeus effect, Fåhraeus-Lindqvist effect, aggregation, disaggregation, stress-induced diffusion

\section{Introduction}

Two of the most striking non-homogeneous effects observed in blood flow in narrow glass tubes are those that bear the names of Fåhraeus (1929) and Fåhraeus \& Lindqvist (1931). The Fåhraeus effect (1929) has to do with the decrease in average concentration of red blood cells (the so-called tube or

$\dagger$ Present address: Department of Mathematical Sciences, Durham University, South Road, Durham DH1 3LE, UK.

$\ddagger$ Corresponding author. Email: owens@dms.umontreal.ca 
dynamic hematocrit $H_{c t}$ ) in human blood as the diameter of the glass tube in which it is flowing decreases. A simple mathematical treatment of the Fåhraeus effect, along the lines of that employed by Sutera et al. (1970), shows that because of migration of cells from the vessel walls towards the tube centre (leading to an average cell speed greater than the average axial velocity component of the surrounding fluid) the tube hematocrit must be less than that of the blood discharged from the end of the tube (the discharge hematocrit). As noted by many authors already, the dynamic decrease in red cell concentration described in the Fåhraeus effect is quite distinct from the possible hematocrit decrease in a small tube due to entry effects (cell screening), although in practice, even in controlled laboratory experiments, both effects may be taking place simultaneously (Gaehtgens et al. (1978)). In the absence of entry effects the discharge hematocrit must equate that of the blood in the reservoir that feeds the tube (the so-called feed hematocrit).

Conjointly with the Fåhraeus effect, and as evidenced by Fåhraeus and Lindqvist in 1931, a decrease in the tube diameter $D$ from approximately $0.3 \mathrm{~mm}$ down to $D \approx 5-7 \mu m$ will result, in healthy human blood, in a drop in the apparent viscosity. This effect, also due to cell migration, results in part from the development of a slippage layer of plasma-rich cell-depleted fluid near the vessel walls. Although the thickness of the cell-depleted lubricating layer depends upon flow rate and tube diameter it seems to do so only weakly and the layer thickness remains more or less in the range $2-4 \mu \mathrm{m}$ (Caro et al. (1978)). Therefore the relative volume of the slippage (low viscosity) layer increases as the tube diameter decreases. This, combined with the Fåhraeus effect, results in a decrease in the apparent viscosity. Thus, although the Fåhraeus effect is important for a correct understanding of the Fåhraeus-Lindqvist effect, it does not entirely explain it (Azelvandre \& Oiknine (1976)), something that we take some trouble to further show in Section 3.4. Enjoying little contestation since their discovery early in the 20th century (although see, for example, Mayer (1965)), the Fåhraeus and Fåhraeus-Lindqvist effects have spawned a huge research literature in the intervening years, and the reader is referred to Blair (1958) and Goldsmith et al. (1989) for readable and fascinating accounts of some of the major research milestones up to the late 1950's and 1980's.

In vivo, the apparent viscosity of blood in the smaller vessels is further reduced because of plasma skimming. That is, a small side branch on a larger vessel may receive blood containing a larger proportion of plasma than in the feeder vessel, due to the fact that the blood supplying it largely 
comes from the near-wall plasma-rich layer (Caro et al. (1978)). It should also be noted, however, that flow resistance in vivo in smaller vessels is larger than is predicted with in vitro data due to the presence of a thick endothelial cell layer (Pries \& Secomb (2005)). For very small vessels $(D \lesssim 5 \mu m)$ both the tube hematocrit and apparent viscosity of normal human blood increase sharply due to the very small clearance between the cells and the vessel walls (Goldsmith et al. 1989; Yen \& Fung 1977).

Although healthy blood under physiological flow conditions in the heart and in the larger arteries of the human cardiovascular system may be adequately described using the Navier-Stokes equations, realistic modelling in the microcirculation requires that proper account be taken of (at the very least) radial variations in viscosity. Macroscopic modelling of blood in narrow tubes has often involved representing the flowing blood as a concentrated core of suspended red blood cells, surrounded by a lower viscosity annulus near the vessel walls. See Secomb (2003) and Sharan \& Popel (2001) and the references therein, for example. Sharan \& Popel (2001) numerically solved a consistent system of nonlinear equations to estimate the effective viscosity in the cell-free layer, the thickness of this layer and the hematocrit in the concentrated core. The system of equations was closed using experimental data for the apparent viscosity and tube hematocrit from Pries et al. (1992). Rather than adopt an empirical macroscopic model a more satisfactory, but potentially hugely more expensive approach, is to directly simulate multi-particle systems. This has been made possible very recently due to the massive increase in computing power available to researchers. Sun \& Munn (2005) have used a two-dimensional lattice Boltzmann method to simulate the flow of both red and white blood cells in $20 \mu \mathrm{m}$ and $40 \mu \mathrm{m}$ conduits at various hematocrits. Both the Fåhraeus and the Fåhraeus-Lindqvist effects could be qualitatively reproduced, although agreement with in vitro experiments was limited due to the authors' use of rigid particles and a channel rather than a tube. Three-dimensional computations using an immersed finite element method and deformable biconcave particles were performed by Liu \& Liu (2006) to investigate the dependence of blood viscosity on shear rate, the influence of cell deformability on the viscosity and the Fåhraeus-Lindqvist effect. The connection between the radial migration of the cells and the Fåhraeus-Lindqvist effect was established. No comparisons were made between experiments and the predicted apparent viscosity with decreasing tube diameter, however. Finally, and most recently, Bagchi (2007) has modelled red blood cells as 
liquid capsules and employed the immersed boundary method to model flows in two-dimensional rectangular channels involving as many as 2500 cells. Comparisons of the predictions of the size of the cell-free layer in channels of different widths were made with the in vitro data of Bugliarello \& Sevilla (1970) and analytical data of Sharan \& Popel (2001) and showed good agreement. Agreement between the numerical results of Bagchi (2007) and the empirical expression of Pries et al. (1992) for the relative apparent viscosity of blood at three different discharge hematocrits was also very close.

The approach that we have adopted thus far in our work has been to derive and use a macroscopic continuum model for blood flow which nevertheless rests on sound microscopic-level foundations (Fang \& Owens 2006; Owens 2006). Owens (2006) showed that predictions of shear stress hysteresis loops in triangular hysteresis experiments in a Couette rheometer were in close agreement with the experimental data of Bureau et al. (1980) and resulted from a complex mixture of shear-thinning, viscoelastic and thixotropic effects. Fang \& Owens (2006) used the microstructure-based constitutive model of Owens (2006) to investigate the steady, oscillatory and pulsatile flow of blood in a tube of radius $0.43 \mathrm{~mm}$. Elastic effects were most pronounced at low flow rates and low flow rate amplitudes and agreement with data from Thurston (1975) was good. In the present paper the basic model is extended to include the possibility of describing highly non-homogeneous flows. In particular, the new developments outlined in the theoretical sections that follow allow us to investigate blood flow in tubes of sufficiently small diameter that wall effects become significant.

The present paper is divided into two major sections. The first, Section 2, deals at some length with the mathematical development of the non-homogeneous model and leads to the coupled nonlinear system of equations $(2.43),(2.45),(2.46),(2.51)$ and $(2.55)$ to be solved in a straight rigid walled tube, subject to an imposed pressure gradient and satisfying the boundary conditions given in Section 2.2.3. The model is derived by starting with the basic equations in a solution of non-interacting dumbbells for the number density and polymeric stress, that are already available in the polymeric fluids literature (Beris \& Mavrantzas 1994; Bhave et al. 1991; Cook \& Rossi 2004; Rossi et al. 2006). As a consequence, these basic equations are simply written down in Section 2.1. The non-homogeneous blood model proposed in this paper conceives of blood as an ensemble of aggregates undergoing binary interactions, each aggregate consisting of different numbers of cells that are modelled by Hookean dumbbells, on the assumption that rates of deformation are small. The modifications that 
have to be brought to bear on the original kinetic theory are clearly explained in Section 2.2. The substantial simplifications that may be made to the full set of non-homogeneous blood equations in the case of steady axisymmetric flow in a straight, rigid walled tube are detailed in Section 2.2.4.

The second major section in the paper is concerned with the numerical solution of the simplified non-homogeneous equations in a tube. Section 3.3 shows how, when the tube hematocrit is fixed, the basic variables vary with the radial distance at different values of the Péclet number and pressure gradient. Next, by allowing the tube hematocrit to vary with tube diameter according to an empirical relation of Pries et al. (1992), we study the influence of both the Fåhraeus effect and other nonhomogeneous effects on the apparent viscosity of steady blood flow in tubes of various diameters.

\section{Mathematical modelling}

\subsection{Basic equations for a solution of non-interacting dumbbells}

Consider an ensemble of non-interacting Hookean dumbbells in a viscous solvent, each dumbbell consisting of two point masses (both of mass $m$ ) joined by a massless linear spring, as shown in Fig. 1. The two point masses are labelled "1" and "2" and have position vectors $\boldsymbol{r}_{1}$ and $\boldsymbol{r}_{2}$, respectively. The position vector of the centre of mass is therefore $\boldsymbol{r}_{c}=\left(\boldsymbol{r}_{1}+\boldsymbol{r}_{2}\right) / 2$ and we denote the end to end vector $\boldsymbol{r}_{2}-\boldsymbol{r}_{1}$ by $\boldsymbol{q}$. The tension $\boldsymbol{F}$ in the spring is calculated from $\boldsymbol{F}=H \boldsymbol{q}$, where $H$ is the spring constant. Suppose that $\boldsymbol{p}$ and $\boldsymbol{P}$ denote, respectively, the momentum vectors associated with the centre of mass and the internal degrees of freedom. Then we define the phase space distribution function $f=f\left(\boldsymbol{r}_{c}, \boldsymbol{q}, \boldsymbol{p}, \boldsymbol{P}, t\right)$ to be such that $f\left(\boldsymbol{r}_{c}, \boldsymbol{q}, \boldsymbol{p}, \boldsymbol{P}, t\right) d \boldsymbol{r}_{c} d \boldsymbol{q} d \boldsymbol{p} d \boldsymbol{P}$ is the number of dumbbells having centre of mass in the range $\left[\boldsymbol{r}_{c}, \boldsymbol{r}_{c}+d \boldsymbol{r}_{c}\right]$, end-to-end vector in the range $[\boldsymbol{q}, \boldsymbol{q}+d \boldsymbol{q}]$ and momenta in the ranges $[\boldsymbol{p}, \boldsymbol{p}+d \boldsymbol{p}]$ and $[\boldsymbol{P}, \boldsymbol{P}+d \boldsymbol{P}]$. Starting with the continuity equation for $f$ (the Liouville equation) it may be shown, using standard arguments in polymer kinetic theory (see, for example, Beris \& Mavrantzas (1994); Bhave et al. (1991); Cook \& Rossi (2004); Rossi et al. (2006)) that if $N$ denotes the number density of dumbbells and if a microscopic length scale $\ell_{0}$ (corresponding to the length in equilibrium of a dumbbell, say) is sufficiently small relative to a macroscopic length scale (a tube radius, for example) then neglecting quantities of third and higher 
order in the ratio of these length scales leads to an equation for $N$ of the form

$$
\frac{D N}{D t}=D_{t r} \nabla^{2} N-\frac{1}{2 \zeta} \nabla \nabla: \tau,
$$

where $D / D t$ denotes the material derivative $\partial / \partial t+\boldsymbol{v} \cdot \nabla$ and $\boldsymbol{v}$ is the fluid velocity. In (2.1) $D_{t r}=$ $k_{B} T / 2 \zeta$ is the translational diffusivity, $k_{B}$ is the Boltzmann constant, $T$ the temperature, $\zeta$ a friction factor and $\tau$ the elastic stress tensor, representing the contribution to the total (Cauchy) stress tensor from the dumbbells. If it is further assumed that $\|\nabla v\| \ll \lambda_{H}^{-1}$, where $\lambda_{H}=\zeta / 4 H$ is the relaxation time for an isolated dumbbell, we may derive an approximate evolution equation for the orientation tensor $\langle\boldsymbol{q} \boldsymbol{q}\rangle$ in the form

$$
\langle\boldsymbol{q} \boldsymbol{q}\rangle=D_{t r} \nabla^{2}\langle\boldsymbol{q} \boldsymbol{q}\rangle+\frac{4 N k_{B} T}{\zeta} \boldsymbol{\delta}-\frac{4 H}{\zeta}\langle\boldsymbol{q} \boldsymbol{q}\rangle
$$

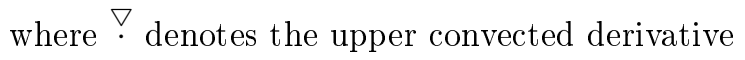

$$
\frac{D \cdot}{D t}-\nabla \boldsymbol{v} \cdot-\cdot \nabla \boldsymbol{v}^{T}
$$

and $\langle\cdot\rangle$ denotes an ensemble average, defined for a quantity $B=B\left(\boldsymbol{r}_{c}, \boldsymbol{q}, \boldsymbol{p}, \boldsymbol{P}, t\right)$ by

$$
\langle B\rangle:=\int_{\mathcal{Q}} \iint_{\mathcal{P}} B f d \boldsymbol{p} d \boldsymbol{P} d \boldsymbol{q} .
$$

In the definition (2.3) above $\mathcal{Q}$ and $\mathcal{P}$ denote, respectively, configuration and momentum space. $\boldsymbol{\delta}$ in (2.2) and throughout this paper denotes the identity tensor.

\subsection{Non-homogeneous blood model}

A healthy unstressed red blood cell assumes a biconcave discoid shape, approximately $8 \mu m$ in diameter and $2-3 \mu m$ maximum thickness and is made up of a viscoelastic membrane filled with an almost saturated solution of haemoglobin. The interested reader is referred to Caro et al. (1978) for further details. Human blood is a suspension of formed elements in plasma, by far the greatest proportion of which consists of red blood cells. It is the behaviour of these cells, therefore, which primarily determines the rheology of blood. In the present paper we attempt to model human blood as a suspension of red cell aggregates in plasma. Although at very low shear rates red cells in healthy human blood form complex networks made up of column-like structures of cells (called rouleaux), as the shear rate increases the rouleaux disassociate from each other. As the shear rate continues to increase the rouleaux progressively fragment and blood becomes a concentrated suspension of single cells (see, for example, Chien \& Jan (1973)). The presence of blood proteins such as fibrinogen and 
immunoglobulins in the plasma is known to play an important role in the process of aggregation (Baskurt \& Meiselman 2003) and the entire disaggregation process is reversible. In the model proposed in the present paper, we consider blood as a concentrated suspension of rouleaux of various sizes, the distribution of rouleaux lengths at any point and time in a flow depending upon the rheological and flow conditions. In the model, each rouleau may undergo binary interactions with others, being able to fragment into two smaller ones or coalesce with another rouleau to form a larger one. Each rouleau will be represented by a Hookean elastic dumbbell, as shown in Fig. 1, and a rouleau composed of $k$ red cells will henceforth be termed a $k$-mer. The number density of $k$-mers will be denoted by $N_{k}$ and if $N_{0}:=\sum_{k=1}^{\infty} k N_{k}$ and $M:=\sum_{k=1}^{\infty} N_{k}$ are the notations used for the number density of red cells and number density of the rouleaux, respectively, it follows that the average rouleau size $n$ at a point in the flow domain is calculated from

$$
n=\frac{N_{0}}{M}
$$

Owens (2006) assumed, as is common in reversible polymer network theory, that aggregation is a Brownian process and that the aggregation rate of $k$-mers is proportional to the equilibrium (no flow, homogeneous) number density $N_{k, 0}$. He also assumed that the fragmentation rate of $k$-mers is proportional to $N_{k}$. We denote the aggregation and fragmentation rate coefficients by $h_{k}$ and $g_{k}$ and will assume a little later that these are functions of the shear rate $\dot{\gamma}$ and time.

The equations equivalent to (2.1) and (2.2) above are now, respectively,

$$
\frac{D N_{k}}{D t}=D_{t r, k} \nabla^{2} N_{k}-\frac{1}{2 \zeta_{k}} \nabla \nabla: \tau_{k}+h_{k} N_{k, 0}-g_{k} N_{k},
$$

and

$$
\langle\boldsymbol{q} \boldsymbol{q}\rangle_{k}=D_{t r, k} \nabla^{2}\langle\boldsymbol{q} \boldsymbol{q}\rangle_{k}+\frac{4\left(k_{B} T+\kappa\right)_{k} N_{k}}{\zeta_{k}} \boldsymbol{\delta}-\frac{4 H}{\zeta_{k}}\langle\boldsymbol{q} \boldsymbol{q}\rangle_{k}+h_{k}\langle\boldsymbol{q} \boldsymbol{q}\rangle_{k, 0}-g_{k}\langle\boldsymbol{q} \boldsymbol{q}\rangle_{k},
$$

where $\zeta_{k}$ is the $k$-dependent friction factor and $D_{t r, k}=\left(k_{B} T+\kappa\right)_{k} / 2 \zeta_{k}$ is the translational (thermal and convective) diffusivity for $k$-mers. The numerator of $D_{t r, k}$ consists of the sum of a very small Brownian contribution and a constant $\kappa$ which takes account of impacts with other blood cells. Since the $k$-mer consists of $k$ cells we choose $\left(k_{B} T+\kappa\right)_{k}=k\left(k_{B} T+\kappa\right)$ and $\zeta_{k}=k \zeta$ and this reduces the diffusion coefficients $D_{t r, k}$ to $D_{t r}=\left(k_{B} T+\kappa\right) / 2 \zeta$ for all $k . \tau_{k}$ in (2.4) denotes the contribution to the total elastic stress tensor $\boldsymbol{\tau}:=\sum_{k=1}^{\infty} \boldsymbol{\tau}_{k}$ from $k$-mers. Substituting from the Kramers expression 
(Kramers (1944)) for the elastic stress tensor $\tau_{k}$ :

$$
\boldsymbol{\tau}_{k}=H\langle\boldsymbol{q} \boldsymbol{q}\rangle_{k}-k N_{k}\left(k_{B} T+\kappa\right) \boldsymbol{\delta},
$$

we then get from (2.4) and (2.5) that

$$
\boldsymbol{\tau}_{k}+\mu_{k}{\stackrel{\nabla}{\boldsymbol{\tau}_{k}}}_{k}-D_{t r} \mu_{k}\left(\nabla^{2} \boldsymbol{\tau}_{k}+\left(\nabla \nabla: \boldsymbol{\tau}_{k}\right) \boldsymbol{\delta}\right)=k N_{k}\left(k_{B} T+\kappa\right) \mu_{k} \dot{\boldsymbol{\gamma}}
$$

where the relaxation time

$$
\mu_{k}=\frac{k \lambda_{H}}{1+g_{k} k \lambda_{H}} .
$$

We see from (2.8) that the relaxation time for a rouleau is increased (through the numerator) relative to that for a single cell due to the increase in friction but decreased (through the denominator) due to the extra relaxation mechanism brought about by fragmentation. With $g_{k}$ a function of shear rate, $\mu_{k}$ is therefore a non-trivial function of both shear rate and rouleau size. Since we expect that in the infinite shear rate limit all rouleaux break up into individual cells with zero aggregation and fragmentation, $\mu_{k}(k \geq 2)$ will tend in this case to 0 and $\mu_{1}$ to $\lambda_{H}$. The constitutive equation satisfied by the elastic stress tensor will then tend to the non-homogeneous Oldroyd B equation

$$
\boldsymbol{\tau}+\lambda_{H} \stackrel{\nabla}{\boldsymbol{\tau}}-D_{t r} \lambda_{H}\left(\nabla^{2} \boldsymbol{\tau}+(\nabla \nabla: \boldsymbol{\tau}) \boldsymbol{\delta}\right)=N_{0}\left(k_{B} T+\kappa\right) \lambda_{H} \dot{\gamma}
$$

As in our previous work (Fang \& Owens 2006; Owens 2006) we choose

$$
\begin{aligned}
g_{k} N_{k} & =\frac{1}{2} \sum_{i=1}^{k-1} F_{i, k-i} N_{k}+\sum_{j=1}^{\infty} K_{k, j} N_{k} N_{j}, \\
h_{k} N_{k, 0} & =\frac{1}{2} \sum_{i=1}^{k-1} K_{i, k-i} N_{i} N_{k-i}+\sum_{j=1}^{\infty} F_{k, j} N_{k+j},
\end{aligned}
$$

where $K_{i, j}$ is an aggregation kernel, expressing the rate at which an $(i+j)$-mer is formed from an $i$-mer and a $j$-mer, and $F_{i, j}$ is a fragmentation kernel, denoting the rate at which an $(i+j)$-mer breaks into an $i$-mer and a $j$-mer. As in Fang \& Owens (2006) and Owens (2006) we choose the aggregation and fragmentation kernels to be simple functions $a$ and $b$ of the shear rate $\dot{\gamma}$ since the reaction sites are principally the end points of the rouleau, independently of $k$. In this case, $g_{k}$ and $h_{k}$ become

$$
\begin{aligned}
g_{k} & =\frac{b(\dot{\gamma})(k-1)}{2}+a(\dot{\gamma}) \sum_{j=1}^{\infty} N_{j}, \\
h_{k} & =\frac{a(\dot{\gamma})}{2 N_{k, 0}} \sum_{i=1}^{k-1} N_{i} N_{k-i}+\frac{b(\dot{\gamma})}{N_{k, 0}} \sum_{j=1}^{\infty} N_{k+j},
\end{aligned}
$$


and we note that, as is to be expected,

$$
\sum_{k=1}^{\infty} k\left(h_{k} N_{k, 0}-g_{k} N_{k}\right)=0,
$$

this expressing the fact (mass conservation) that in any material volume the number of red cells remains constant.

Rather than work with a multi-mode model, which even with a truncated sum $\sum_{k} \tau_{k}$ would necessitate the solution of a potentially large number of constitutive equations of the type (2.7), we compute an average relaxation time $\bar{\mu}:=\mu_{n}$ and may now sum $k$ times $(2.4)$ from $k=1$ to $\infty$ to get

$$
\frac{D N_{0}}{D t}=D_{t r} \nabla^{2} N_{0}-\frac{D_{t r}}{\left(k_{B} T+\kappa\right)} \nabla \nabla: \tau,
$$

and sum (2.7) from $k=1$ to $\infty$, which gives us

$$
\boldsymbol{\tau}+\bar{\mu} \stackrel{\nabla}{\boldsymbol{\tau}}-D_{t r} \bar{\mu}\left(\nabla^{2} \boldsymbol{\tau}+(\nabla \nabla: \boldsymbol{\tau}) \boldsymbol{\delta}\right)=N_{0}\left(k_{B} T+\kappa\right) \bar{\mu} \dot{\gamma}
$$

With the choice $K_{i j}=a(\dot{\gamma})$ and $F_{i j}=b(\dot{\gamma})$ we sum $(2.4)$ from $k=1$ to $\infty$ to get the evolution equation for the number density of aggregates

$$
\frac{D M}{D t}=D_{t r} \nabla^{2} M-\frac{D_{t r}}{\left(k_{B} T+\kappa\right)} \nabla \nabla: \sigma-\frac{a(\dot{\gamma})}{2} M^{2}+\frac{b(\dot{\gamma})}{2}\left(N_{0}-M\right),
$$

where

$$
\boldsymbol{\sigma}=\sum_{k=1}^{\infty} \frac{\boldsymbol{\tau}_{k}}{k}
$$

and satisfies

$$
\boldsymbol{\sigma}+\bar{\mu} \stackrel{\nabla}{\boldsymbol{\sigma}}-D_{t r} \bar{\mu}\left(\nabla^{2} \boldsymbol{\sigma}+(\nabla \nabla: \boldsymbol{\sigma}) \boldsymbol{\delta}\right)=M\left(k_{B} T+\kappa\right) \bar{\mu} \dot{\gamma}
$$

To close the system of equations for $\left(\boldsymbol{v}, p, N_{0}, M, \boldsymbol{\sigma}, \boldsymbol{\tau}\right)$ we must add the usual equation of conservation of linear momentum and the incompressibility constraint:

$$
\begin{aligned}
\rho_{f} \frac{D \boldsymbol{v}}{D t} & =-\nabla p+\eta_{N} \nabla^{2} \boldsymbol{v}+\nabla \cdot \boldsymbol{\tau}, \\
\nabla \cdot \boldsymbol{v} & =0
\end{aligned}
$$

where $\rho_{f}$ denotes the fluid density, $p$ the pressure and $\eta_{N}$ is the constant plasma viscosity.

If $\mathcal{D} \subset \mathbb{R}^{3}$ denotes the region occupied by a blood sample and $V_{e}$ is the volume of a single red 
blood cell (the so-called mean corpuscular volume $\approx 90 \mu \mathrm{m}^{3}$ ), the tube hematocrit $H_{c t}$ is defined as

$$
H_{c t}=\frac{V_{e}}{\operatorname{vol} \mathcal{D}} \int_{\mathcal{D}} N_{0}(\boldsymbol{x}, t) d \boldsymbol{x}
$$

We may also define an average number density of red cells $N_{a v}$ in $\mathcal{D}$ :

$$
N_{a v}:=\frac{1}{\operatorname{vol} \mathcal{D}} \int_{\mathcal{D}} N_{0}(\boldsymbol{x}, t) d \boldsymbol{x}
$$

which, from (2.19) is seen to be related to the hematocrit by $N_{a v}=H_{c t} / V_{e}$.

\subsubsection{Non-dimensionalization of the equations}

We scale the variables as follows:

$$
\begin{array}{r}
\hat{N}_{0}=\frac{N_{0}}{N_{a v}}, \quad \hat{M}=\frac{M}{N_{a v}}, \quad \hat{p}=\frac{p}{N_{a v}\left(k_{B} T+\kappa\right)}, \quad \hat{t}=\frac{t V}{R}, \\
\hat{\boldsymbol{x}}=\frac{\boldsymbol{x}}{R}, \quad \hat{\boldsymbol{v}}=\frac{\boldsymbol{v}}{V}, \quad \hat{\boldsymbol{\tau}}=\frac{\boldsymbol{\tau}}{N_{a v}\left(k_{B} T+\kappa\right)}, \quad \hat{\boldsymbol{\sigma}}=\frac{\boldsymbol{\sigma}}{N_{a v}\left(k_{B} T+\kappa\right)},
\end{array}
$$

where $N_{a v}$ is given by (2.20), $R$ denotes a characteristic length and $V$ a characteristic flow speed (a maximum or average value, for example).

Introducing the non-dimensionalized variables into (2.13)-(2.16) and using the fact that $\bar{\mu}=\mu_{n}$, we get

$$
\frac{D \hat{N}_{0}}{D \hat{t}}=\frac{1}{P e} \hat{\nabla}^{2} \hat{N}_{0}-\frac{1}{P e} \hat{\nabla} \hat{\nabla}: \hat{\boldsymbol{\tau}}
$$

and

$$
\hat{\boldsymbol{\tau}}+D e \stackrel{\nabla}{\hat{\boldsymbol{\tau}}}-\frac{D e}{P e}\left(\hat{\nabla}^{2} \hat{\boldsymbol{\tau}}+(\hat{\nabla} \hat{\nabla}: \hat{\boldsymbol{\tau}}) \boldsymbol{\delta}\right)=\hat{N}_{0} D e \hat{\dot{\gamma}}
$$

In (2.22) we define the (shear rate and time-dependent) Deborah number $D e$ as

$$
D e:=\frac{\bar{\mu} V}{R}=\frac{n D e_{\infty}}{1+g_{n} n D e_{\infty}}
$$

where

$$
D e_{\infty}=\frac{\lambda_{H} V}{R}
$$

The Péclet number

$$
P e:=\frac{V R}{D_{t r}}=\frac{2 \zeta R V}{\left(k_{B} T+\kappa\right)},
$$

relates the rate of convection of the flow to the rate of mass diffusion. The physical significance of the ratio $D e_{\infty} / P e$ may be seen by computing

$$
\frac{D e_{\infty}}{P e}=\frac{\lambda_{H} V}{R} \frac{\left(k_{B} T+\kappa\right)}{2 \zeta R V}=\frac{1}{8} \frac{\left(k_{B} T+\kappa\right)}{H R^{2}}=\frac{\ell_{0}^{2}}{24 R^{2}},
$$


where $\ell_{0}=\sqrt{3\left(k_{B} T+\kappa\right) / H}$ is the ensemble average equilibrium length of a simple $(k=1)$ Hookean dumbbell in the absence of wall effects. $\left(D e_{\infty} / P e\right)^{1 / 2}$ therefore measures the relative magnitudes of a microscopic length scale to a macroscopic length scale. Where this ratio is non-negligible wall effects may be considered to be important. Moyers-Gonzalez \& Owens (2008) show that when $D e_{\infty} / P e \ll 1$ boundary layers of thickness $O\left(D e_{\infty} / P e\right)^{1 / 2}$ develop.

The non-dimensionalized equations for $\hat{M}$ and $\hat{\boldsymbol{\sigma}}$ read

$$
\begin{gathered}
\frac{D \hat{M}}{D \hat{t}}=\frac{1}{P e} \hat{\nabla}^{2} \hat{M}-\frac{1}{P e} \hat{\nabla} \hat{\nabla}: \hat{\boldsymbol{\sigma}}-\frac{\hat{a}(\hat{\dot{\gamma}})}{2} \hat{M}^{2}+\frac{\hat{b}(\hat{\dot{\gamma}})}{2}\left(\hat{N}_{0}-\hat{M}\right), \\
\hat{\boldsymbol{\sigma}}+D e \hat{\boldsymbol{\sigma}}-\frac{D e}{P e}\left(\hat{\nabla}^{2} \hat{\boldsymbol{\sigma}}+(\hat{\nabla} \hat{\nabla}: \hat{\boldsymbol{\sigma}}) \boldsymbol{\delta}\right)=\hat{M} D e \hat{\dot{\boldsymbol{\gamma}}}
\end{gathered}
$$

where

$$
\hat{a}(\hat{\dot{\gamma}}):=a(\dot{\gamma}) N_{a v} \frac{R}{V}=a(\dot{\gamma}) N_{a v} \frac{\lambda_{H}}{D e_{\infty}} \text {, and } \hat{b}(\hat{\gamma}):=b(\dot{\gamma}) \frac{R}{V}=b(\dot{\gamma}) \frac{\lambda_{H}}{D e_{\infty}},
$$

are non-dimensional aggregation and fragmentation rates, respectively. The precise functional forms of $a(\dot{\gamma}) N_{a v}$ and of $b(\dot{\gamma})$ are determined from experiments (see Murata \& Secomb 1988; Shiga et al. 1983) and from the $a-b$ relationship in steady homogeneous flow, respectively. See Appendix A. As before, the average aggregate size is $n=\hat{N}_{0} / \hat{M}$. If we introduce

$$
\eta_{\infty}:=N_{a v}\left(k_{B} T+\kappa\right) \lambda_{H} \text { and } \eta:=\frac{D e_{\infty} \eta_{N}}{\eta_{\infty}}
$$

then the non-dimensionalized equations of motion become

$$
\begin{aligned}
R e \frac{D \hat{\boldsymbol{v}}}{D \hat{t}} & =-\hat{\nabla} \hat{p}+\eta \hat{\nabla}^{2} \hat{\boldsymbol{v}}+\hat{\nabla} \cdot \hat{\boldsymbol{\tau}}, \\
\hat{\nabla} \cdot \hat{\boldsymbol{v}} & =0,
\end{aligned}
$$

with the Reynolds number Re being defined as

$$
R e=\frac{\rho_{f} V R D e_{\infty}}{\eta_{\infty}}
$$

(Note that, as an alternative to the Reynolds number defined above, scaling time with $\lambda_{H}$ rather than $R / V$ would lead to a more conventional definition of a Reynolds number: $R e=\rho_{f} V R / \eta_{\infty}$.)

For the rest of the paper, and for the sake of simplicity, we will drop the hats on the nondimensional variables. 


\subsubsection{Equations for axisymmetric non-homogeneous flow of blood in a uniform pipe}

We introduce cylindrical polar coordinates $(r, \theta, z)$ and consider steady pressure-driven axisymmetric flow in a uniform pipe of radius $R$ having axis in the $z$-direction. Then, seeking a solution with a velocity field of the form $\boldsymbol{v}=\left(0,0, v_{z}(r)\right)$, pressure $p=p(z)$ and all other variables functions of $r$ alone, the equations (2.21)-(2.27) may be written as

$$
\begin{aligned}
& \frac{1}{r} \frac{d}{d r}\left(r \frac{d N_{0}}{d r}\right)-\left(\frac{1}{r} \frac{d}{d r}\left(r \frac{d \tau_{r r}}{d r}\right)+\frac{1}{r} \frac{d}{d r}\left(\tau_{r r}-\tau_{\theta \theta}\right)\right)=0 \\
& \frac{1}{P e} \frac{1}{r} \frac{d}{d r}\left(r \frac{d M}{d r}\right)-\frac{1}{P e}\left(\frac{1}{r} \frac{d}{d r}\left(r \frac{d \sigma_{r r}}{d r}\right)+\frac{1}{r} \frac{d}{d r}\left(\sigma_{r r}-\sigma_{\theta \theta}\right)\right)-\frac{a N_{0}}{2 n} M+\frac{b}{2}\left(N_{0}-M\right)=0 \\
& \tau_{r r}-\frac{D e}{P e}\left(\frac{2}{r} \frac{d}{d r}\left(r \frac{d \tau_{r r}}{d r}\right)+\frac{1}{r} \frac{d}{d r}\left(\tau_{r r}-\tau_{\theta \theta}\right)-\frac{2}{r^{2}}\left(\tau_{r r}-\tau_{\theta \theta}\right)\right)=0 \\
& \tau_{\theta \theta}-\frac{D e}{P e}\left(\frac{1}{r} \frac{d}{d r}\left(r \frac{d \tau_{\theta \theta}}{d r}\right)+\frac{1}{r} \frac{d}{d r}\left(r \frac{d \tau_{r r}}{d r}\right)+\frac{1}{r} \frac{d}{d r}\left(\tau_{r r}-\tau_{\theta \theta}\right)+\frac{2}{r^{2}}\left(\tau_{r r}-\tau_{\theta \theta}\right)\right)=0 \\
& \tau_{r z}-D e \frac{d v_{z}}{d r} \tau_{r r}-\frac{D e}{P e}\left(\frac{1}{r} \frac{d}{d r}\left(r \frac{d \tau_{r z}}{d r}\right)-\frac{\tau_{r z}}{r^{2}}\right)-N_{0} D e \frac{d v_{z}}{d r}=0 \\
& \quad-\frac{D p}{d z}+\eta \frac{1}{r} \frac{d}{d r}\left(r \frac{d v_{z}}{d r}\right)+\frac{1}{r} \frac{d}{d r}\left(r \tau_{r z}\right)=0 \\
& \left.\tau_{z z}-2 D e \frac{d v_{z}}{d r} \tau_{r z}-\frac{d}{P e}\left(r \frac{d \tau_{z z}}{d r}\right)+\frac{1}{r} \frac{d}{d r}\left(r \frac{d \tau_{r r}}{d r}\right)+\frac{1}{r} \frac{d}{d r}\left(\tau_{r r}-\tau_{\theta \theta}\right)\right)=0
\end{aligned}
$$

Since $v_{z}=v_{z}(r)$, the incompressibility condition (2.28) is identically satisfied. $\tau_{r \theta}, \tau_{\theta r}, \tau_{z \theta}, \tau_{\theta z}$ and the corresponding components of $\boldsymbol{\sigma}$ are all zero. Equations for the remaining components of $\boldsymbol{\sigma}$ are analogous to those for the corresponding components of $\tau$ and are obtained by replacing $\tau_{i j}$ with $\sigma_{i j}$ and $N_{0}$ with $M$ in (2.31)-(2.34).

\subsubsection{Boundary conditions}

We assume, as have other authors before us (Bhave et al. 1991; Cook \& Rossi 2004; Rossi et al. 2006), that the principal axes of the microstructure on the wall are orientated in the $z$-direction, so that non-dimensionalization of the Kramers expression (2.6) then leads to the elastic stress boundary condition

$$
\boldsymbol{\tau}=N_{0} Q_{0}^{2} \boldsymbol{e}_{z} \boldsymbol{e}_{z}-N_{0} \boldsymbol{\delta}
$$


where $Q_{0}$ is the length of a Hookean dumbbell divided by $\ell_{0} / \sqrt{3}$ and $\boldsymbol{e}_{z}$ is a unit vector in the $z$-direction. Since $\tau_{z z}$ is not required in order to solve the equations (2.29)-(2.33) and (2.35) for the other variables, we have no need to estimate $Q_{0}$ and the wall stress condition on the remaining components is just $\tau_{i j}=-N_{0} \delta_{i j}$. Along the axis of symmetry $\tau_{r z}$ is set equal to zero and the normal derivatives of $\tau_{r r}$ and $\tau_{\theta \theta}$ equal zero, from symmetry considerations. Boundary conditions for $\boldsymbol{\sigma}$ are equivalent to the ones for $\tau$, the only difference being that $N_{0}$ is exchanged for $M$.

Subtracting (2.31) and (2.32) we get

$$
\tau_{r r}-\tau_{\theta \theta}-\frac{D e}{P e}\left(\frac{1}{r} \frac{d}{d r}\left(r \frac{d \tau_{r r}}{d r}\right)-\frac{1}{r} \frac{d}{d r}\left(r \frac{d \tau_{\theta \theta}}{d r}\right)-\frac{4}{r^{2}}\left(\tau_{r r}-\tau_{\theta \theta}\right)\right)=0 .
$$

Now, let $w=\tau_{r r}-\tau_{\theta \theta}$. Then (2.37) is equal to

$$
w-\frac{D e}{P e}\left(\frac{1}{r} \frac{d}{d r}\left(r \frac{d w}{d r}\right)-4 \frac{w}{r^{2}}\right)=0
$$

together with the homogeneous boundary conditions

$$
\begin{gathered}
\frac{d w}{d r}=0 \text { at } r=0, \\
w=0 \text { at } r=1 .
\end{gathered}
$$

The solution to (2.38) with boundary conditions (2.39)-(2.40) is $w \equiv 0$, which implies $\tau_{r r} \equiv \tau_{\theta \theta}$. Using the same arguments with the corresponding equations for $\sigma_{r r}$ and $\sigma_{\theta \theta}$ allows us to conclude that $\sigma_{r r} \equiv \sigma_{\theta \theta}$.

$N_{0}$ and $M$ satisfy the natural boundary conditions

$$
\frac{d N_{0}}{d r}-\frac{d \tau_{r r}}{d r}=0
$$

and

$$
\frac{d M}{d r}-\frac{d \sigma_{r r}}{d r}=0
$$

at the walls $r=1$. Equation (2.41) is equivalent to ensuring that there is no flux of rouleaux through the tube wall. In addition, the non-dimensional form of (2.20) where the region $\mathcal{D}$ is taken to be any right circular cylindrical section of the tube, imposes the condition

$$
\int_{0}^{1} N_{0} r \mathrm{~d} r=\frac{1}{2}
$$

upon $N_{0}$. Solvability of (2.30) for $M$ requires that $M$ be bounded at $r=0$ and this is achieved in the weak finite element formulation (see Section 3.2) by setting $\partial M / \partial r=0$ on the axis of symmetry. 


\subsubsection{Further simplifications}

Heeding the arguments above, it may be seen that the set of equations describing steady axisymmetric tube flow reduces to

$$
\begin{gathered}
\frac{1}{r} \frac{d}{d r}\left(r \frac{d N_{0}}{d r}\right)-\frac{1}{r} \frac{d}{d r}\left(r \frac{d \tau_{r r}}{d r}\right)=0 \\
\frac{1}{P e} \frac{1}{r} \frac{d}{d r}\left(r \frac{d M}{d r}\right)-\frac{1}{P e}\left(\frac{1}{r} \frac{d}{d r}\left(r \frac{d \sigma_{r r}}{d r}\right)\right)-\frac{a N_{0}}{2 n} M+\frac{b}{2}\left(N_{0}-M\right)=0 \\
\tau_{r r}-\frac{D e}{P e}\left(\frac{2}{r} \frac{d}{d r}\left(r \frac{d \tau_{r r}}{d r}\right)\right)=0 \\
\tau_{r z}-D e \frac{d v_{z}}{d r} \tau_{r r}-\frac{D e}{P e}\left(\frac{1}{r} \frac{d}{d r}\left(r \frac{d \tau_{r z}}{d r}\right)-\frac{\tau_{r z}}{r^{2}}\right)-N_{0} D e \frac{d v_{z}}{d r}=0 \\
\tau_{z z}-2 D e \frac{d v_{z}}{d r} \tau_{r z}-\frac{D e}{P e}\left(\frac{1}{r} \frac{d}{d r}\left(r \frac{d \tau_{z z}}{d r}\right)+\frac{1}{r} \frac{d}{d r}\left(r \frac{d \tau_{r r}}{d r}\right)\right)=0 \\
-\frac{d p}{d z}+\eta \frac{1}{r} \frac{d}{d r}\left(r \frac{d v_{z}}{d r}\right)+\frac{1}{r} \frac{d}{d r}\left(r \tau_{r z}\right)=0
\end{gathered}
$$

In the paragraphs that follow we demonstrate that yet further simplification is possible. We begin by multiplying $(2.44)$ by an arbitrary function $\phi \in C^{\infty}(0,1)$ and integrating by parts over $[0,1]$ to get

$$
-\int_{0}^{1} r \frac{d N_{0}}{d r} \frac{d \phi}{d r} d r+\left.\frac{d N_{0}}{d r}\right|_{r=1}=-\int_{0}^{1} r \frac{d \tau_{r r}}{d r} \frac{d \phi}{d r} d r+\left.\frac{d \tau_{r r}}{d r}\right|_{r=1}
$$

Applying (2.41) we have,

$$
\int r \frac{d N_{0}}{d r} \frac{d \phi}{d r} \mathrm{~d} r=\int r \frac{d \tau_{r r}}{d r} \frac{d \phi}{d r} \mathrm{~d} r
$$

Since (2.50) holds for any $\phi \in C^{\infty}(\Omega)$, we get

$$
\frac{d N_{0}}{d r}=\frac{d \tau_{r r}}{d r}
$$

so that integrating yields

$$
N_{0}=\tau_{r r}+C
$$

for some constant $C$, chosen such that (2.43) is satisfied. Eqn. (2.51) expresses the original idea of Onuki (2002) that the degree of inhomogeneity in a polymer solution due to stress-induced migration is proportional to the first normal stress difference in the flow.

Letting $\mathbf{T}:=\eta \dot{\gamma}+\tau$, we can write the equation of motion, (2.49) as

$$
\frac{1}{r} \frac{d}{d r}\left(r T_{r z}\right)=\frac{d p}{d z}
$$


where

$$
T_{r z}=\eta \frac{d v_{z}}{d r}+\tau_{r z}
$$

We solve for $T_{r z}$ in (2.52), and noting that the shear stress should be bounded at $r=0$ we have,

$$
T_{r z}=\frac{r}{2} \frac{d p}{d z}
$$

Substituting (2.53) into (2.47) leads to

$$
\eta \frac{D e}{P e}\left(\frac{1}{r} \frac{d}{d r}\left(r \frac{d}{d r}\left(\frac{d v_{z}}{d r}\right)\right)-\frac{1}{r^{2}} \frac{d v_{z}}{d r}\right)-\frac{d v_{z}}{d r}\left(D e \tau_{r r}+N_{0} D e+\eta\right)+T_{r z}=0 .
$$

This is a differential equation for $d v_{z} / d r$, with boundary conditions

$$
\frac{d v_{z}}{d r}=0 \text { at } r=0
$$

i.e., zero shear rate along the line of symmetry and

$$
\frac{d v_{z}}{d r}=\frac{1}{2 \eta} \frac{d p}{d z} \text { at } r=1 .
$$

Boundary condition (2.57) may require further explanation. We note that from (2.36) that $\tau_{r z}$ is zero at the wall, and this implies, from (2.53), that at $r=1$

$$
\frac{d v_{z}}{d r}=\frac{T_{r z}}{\eta}
$$

Using (2.54) we now get (2.57).

\section{Numerical Results}

In this section we present the results of solving (2.45), (2.46) (and the corresponding equation for $\sigma_{r r}$ ) and (2.55), subject to an imposed (dimensional) pressure gradient $P$ and satisfying the boundary conditions on $M, \tau_{r r}, \sigma_{r r}$ and $d v_{z} / d r$ described in the paragraphs above. $N_{0}$ is found from (2.43) and (2.51). Once converged solutions have been found for $\tau_{r r}$ and $d v_{z} / d r$, one may compute $\tau_{z z}$ and $v_{z}$, should one wish to do so, from (2.48) and the identity

$$
v_{z}(r)=-\int_{r}^{1} \frac{d v_{z}}{d r^{\prime}} d r^{\prime}
$$

\subsection{Parameter selection}

Since the fragmentation rate function $b(\dot{\gamma})$ is determined from the $a-b$ relationship that holds in steady homogeneous flow (see Appendix A), we need to select the parameters that appear in the 
Parameter

plasma viscosity

zero shear rate polymeric viscosity

high shear rate polymeric viscosity

Cross model power law exponent

Cross model parameter

Maxwell relaxation time

infinite shear rate Deborah number

homogeneous average cell number density

$\begin{array}{ccc}\text { symbol } & \text { assigned value } & \text { units } \\ \eta_{N} & 0.001 & \text { Pa.s } \\ \eta_{0} & 0.0326 & \text { Pa.s } \\ \eta_{\infty} & 0.00234 & \text { Pa.s } \\ \mathrm{m} & 2.1238 & - \\ \beta & 0.7014 & \mathrm{~s}^{m} \\ \lambda_{H}=\zeta / 4 H & 0.005 & \mathrm{~s} \\ D e_{\infty}=\lambda_{H} V / R & 1.4 & - \\ N_{a v, h o m} & 5 \times 10^{15} & \mathrm{~m}^{-3}\end{array}$

TABLE 1. Material and flow parameters and their assigned values

Cross model description (A 2) of the contribution of the cell suspension to the steady shear viscosity (the other contribution is that due to the plasma). We shall refer to this contribution as the steady polymeric shear viscosity. The parameters required by (A 2) are the zero shear-rate steady polymeric shear viscosity $\eta_{0}$, the infinite shear-rate steady polymeric shear viscosity $\eta_{\infty}$, and parameters $\beta$ and $m$ (a power law exponent), that determine the shape of the viscosity curve as a function of shear rate as it passes between $\eta_{0}$ and $\eta_{\infty}$. The values of the aforementioned parameters are tabulated in Table 1 and for a justification of the value assigned to $\eta_{\infty}$, see Section 3.4. The plasma viscosity $\eta_{N}$ is set equal to 0.001Pa.s.

With $\lambda_{H}$ chosen as $0.005 \mathrm{~s}$ (see Moyers-Gonzalez et al. (2008), where this value was best for agreement with the small amplitude oscillatory data of Thurston (1975)) and the homogeneous (infinite radius tube) average cell number density $N_{a v, h o m}$ (see Appendix A) selected to be equal to $5 \times 10^{6}$ per $\mu \ell$ (a realistic value, for an hematocrit of 0.45 ), the formula (2.26) for $\eta_{\infty}$ now fixes the value of $k_{B} T+\kappa$. We note, in passing, that this necessitates that $\kappa$ be several orders of magnitude greater than $k_{B} T$ and this is consistent with the experimental observation by Goldsmith \& Marlow (1979), for example, that the principal cause of diffusion is that due to particle-particle interactions rather than thermal effects. $D e_{\infty}$ was chosen equal to 1.4 (this being the product of $\lambda_{H}$ with a shear rate of $280 \mathrm{~s}^{-1}$, characteristic of that found in an arteriole (Caro et al. (1978))). The Péclet number 
$P e$ is determined from $R^{2}$ from

$$
P e=\frac{V R}{D_{t r}}=\frac{2 \zeta R V}{\left(k_{B} T+\kappa\right)}=\left(\frac{2 \zeta D e_{\infty}}{\left(k_{B} T+\kappa\right) \lambda_{H}}\right) R^{2}=\left(\frac{8 D e_{\infty} H}{\left(k_{B} T+\kappa\right)}\right) R^{2}
$$

and best results were found when the ratio between the two quantities was set equal to $1.75 \times 10^{11}$.

This results in a characteristic single red cell dimension of $\sqrt{3\left(k_{B} T+\kappa\right) / H}=1.38 \times 10^{-5} \mathrm{~m}$ which is slightly on the high side. However, it is not expected that increasing the ratio between the Péclet number and $R^{2}$ so as to reduce the cell dimension to $O\left(10^{-6}\right)$ will significantly change the presented results. Finally, in order to be able to impose a given physical (dimensional) pressure gradient $P$ (say) we calculate the dimensionless pressure drop from

$$
\frac{d p}{d z}=\left(\frac{R}{N_{a v}\left(k_{B} T+\kappa\right)}\right) P=\left(\frac{R \lambda_{H}}{\eta_{\infty}}\right) P .
$$

\subsection{Numerical method}

The system of equations is discretized using a standard second-order Galerkin finite element method and the resulting nonlinear system is solved iteratively with a Picard-type iteration. Starting with an initial guess for the velocity gradient (calculated by assuming simple Poiseuille flow in a pipe subject to the imposed $P$ ) we perform two inner loops: the first for the $\tau_{r r}-N_{0}$ pair (Eqns. (2.43), (2.46) and (2.51)) and the second for the $\sigma_{r r}-M$ pair (Eqn. (2.45) and the $\sigma_{r r}$ equation). The variables in each inner loop are considered to have converged when the $L^{2}$ norm of the difference in successive iterates drops below a prescribed tolerance (equal to $\varepsilon=1 \times 10^{-4}$ ). $d v_{z} / d r$ is then updated and the process continued until the $L^{2}$ norm of the difference in successive iterative values of all variables is less than $\varepsilon$. In order to accurately approximate the solution in the boundary layer and near the axis of symmetry the finite element mesh was refined in $r \in[0,0.05] \bigcup[0.95,1]$. In the main part of the mesh the element length $\Delta r$ was set equal to $5 \times 10^{-3}$ and in the refined regions to one tenth of this value. We experimented with different $\varepsilon$ and $\Delta r$ but no obvious difference was to be seen in the computed solutions by reducing either any further, at least for the parameter set given in Table 1.

Although we defer a complete asymptotic analysis until Part II of this paper, it may be seen that as $P e \rightarrow \infty$ the dominant terms in Eqn. (2.45) in those parts of the normalized flow domain $[0,1]$ 
where

$$
\frac{1}{r} \frac{d}{d r}\left(r \frac{d M}{d r}\right) \text { and } \frac{1}{r} \frac{d}{d r}\left(r \frac{d \sigma_{r r}}{d r}\right)
$$

remain finite will be

$$
-\frac{a N_{0}}{2 n} M+\frac{b}{2}\left(N_{0}-M\right)
$$

and that therefore the relationship between $M$ and $N_{0}$ there will assume the same form as that for homogeneous flow (cf. Eqn. (9) of Owens (2006)). In the boundary layer adjacent to the tube wall steep derivatives develop in $M$ so that the dominant balance as $P e \rightarrow \infty$ changes and the method of matched asymptotic expansions is needed in order to correctly match the inner to the outer solution. Equally, as explained at greater length by Moyers-Gonzalez \& Owens (2008) in Part II of this paper, the development of a steep internal layer in the steady homogeneous average aggregate size and upon which the function $b$ (and therefore $M$ ) depends will mean that the $M$ will no longer behave as in (3.3) as $P e \rightarrow \infty$ and sufficiently close to $r=0$. In Fig. 2(a) we show, as a check on our numerical scheme, the solution $M$ to (2.45) and the homogeneous solution, when $P e=85750$ and the physical pressure gradient is $P=-1000 \mathrm{Nm}^{-3}$. The agreement between the two solutions is excellent in a region in the interior of the flow domain, as anticipated, the only remaining discrepancy being due to the fact that sufficiently close to the axis of symmetry, $M$, in the non-homogeneous case, is larger than in the homogeneous case. In Fig. 2(b) we show the non-homogeneous boundary layer solution. We will comment on the behaviour of $M$ as $P e$ and $P$ are allowed to vary in the following section.

\subsection{Results with varying $P e$ and $P$}

In Figs. 3-6 we show how the computed flow variables change as a function of normalized radial distance as the Péclet number $P e$ increases from $4.38 \times 10^{2}$ (corresponding to a tube of physical radius $50 \mu \mathrm{m}$ ) to $4.38 \times 10^{4}$ (for which the equivalent tube radius is $R=500 \mu \mathrm{m}$ ) and as the physical pressure gradient $P$ passes from $-10 \mathrm{Nm}^{-3}$ to $-1000 \mathrm{Nm}^{-3}$. The results are most easily considered by dividing them into two groups: those at $P e=4.38 \times 10^{2}$ and those at the higher Péclet numbers. We begin our comments on the results with those at the two highest Péclet numbers. In Figs. 1114 we have produced surface plots of $N_{0}, M, n$ and $N_{0} D e$ to show how these quantities vary as functions of the normalized radial distance and Péclet number when the pressure gradient $P$ is fixed at $-500 \mathrm{Nm}^{-3}$. All results in Sections 3.3.1 and 3.3.2 have been computed at a fixed tube hematocrit 
equal to the feed/discharge hematocrit. The results of more realistic calculations, where we allow $H_{c t}$ to decrease with tube diameter, are reported in Section 3.4.

3.3.1. $P e=1.09 \times 10^{4}$ and $4.38 \times 10^{4}$.

Variations in $N_{0}$ with $r$ as the Péclet number is reduced (equivalently, as the tube radius $R$ is reduced) and visible in Figs. 3-4 and Fig. 11, indicate migration of the cells away from the walls. The process of diffusion is driven by the anisotropy of $\tau_{r r}$, and indeed would continue to be even in equilibrium $(P=0)$. It is worth mentioning in passing that if the model were not viscoelastic $\left(\lambda_{H}=0\right) \tau_{r r}$ would, of course, be zero and there would be no particle migration. Migration effects in the present steady flow, where inertial effects are absent, are therefore dependent on the deformability of the cells and leave a cell-depleted region near the tube wall. In the infinite Pe case (homogeneous flow) wall effects would be absent and $N_{0}=1$, but as the Péclet number is reduced the near-wall number density $N_{0}$ decreases and the boundary layer thickens. Since mass conservation requires that (2.43) be satisfied, a smaller cell number density near the walls is compensated for with a higher density near the axis of symmetry as cells diffuse across fluid streamlines towards the centre of the tube. In this way, wall effects are felt by the fluid throughout the flow domain, however large the Péclet number and however thin the boundary layer. An increasing flow rate (and corresponding shear rate) leads to closer alignment of the rouleaux with the streamlines and an increase in stretch in this direction. As evidenced by Figs 3-6, these kinematical and microstructural changes lead to a reduction in $\left|\tau_{r r}\right|$ at any radial position and to an increase in $\left|\tau_{r z}\right|$. The consequence of normal stresses having smaller magnitude is a reduction in migration effects.

As the pressure drop increases, so for a tube of any radius (fixed $P e$ ) the volume flow rate and the maximum shear rate (attained at the walls) increase (see Figs. 7-8). Figs. 7-8 also indicate that the centreline axial velocity value and wall shear rates increase with Péclet number. At the lowest flow rate $\left(P=-10 \mathrm{Nm}^{-3}\right)$, the shear rate in the bulk flow (outside the boundary layers) is extremely small and as a result aggregates are not broken up in any great numbers. This means (see Fig. 3) that the number density of aggregates $M$ is small and uniform in the bulk flow which in turn means that $n$ is larger than the values it assumes at the same radial positions at much higher flow rates 
(compare Figs. 9 and 10, for example). Fig. 12 shows the behaviour of $M$ as a function of $P e$ and $r$, calculated with an intermediate pressure gradient $P=-500 \mathrm{Nm}^{-3}$.

At the largest Péclet number, the impact on the viscosity $N_{0} D e$ of nearly constant $N_{0}$ and $n$ in the bulk flow (and away from the centreline, in the case of $P=-1000 \mathrm{Nm}^{-3}$ ) is that it too shows only small variations with $r$ in this region of the flow and, as a consequence, the velocity profile is essentially parabolic and the elastic shear stress $\tau_{r z}$ a linear function of $r$ (Fig. 5). With increasing flow rates the shear rate increases at any radial position $r$ (Figs. 7-8), aggregates are broken up and all the more so as one approaches the wall. This leads inevitably to profiles of $n$ and $N_{0} D e$ that are monotonic decreasing functions of $r$ at any given flow rate, whose average values decrease with increasing flow rates. As the flow rate increases to the maximum value considered in our numerical experiments (Fig. 10), aggregates have been broken up at all Péclet numbers to the extent that the average aggregate size is only slightly greater than 1 away from the centreline.

3.3.2. $P e=4.38 \times 10^{2}$

From Figs. 3-6 it may be observed that at a Péclet number of $4.38 \times 10^{2}$ cell migration is at its strongest, in response to the greater elastic normal stress effects near the walls and the deeper penetration of these effects into the flow domain compared with those at the Péclet numbers considered in Section 3.3.1. Although, of the three values shown in Fig. 3, the centreline value of the number density $N_{0}$ is found to be a maximum for the narrowest tube $(R=50 \mu \mathrm{m})$, the corresponding number density of aggregates there is sufficiently large compared to its value for tubes of larger radius that the variation of the average aggregate size $n$ along the centreline as the tube radius is reduced from $500 \mu m$ to $50 \mu m$ is non-monotonic. The same non-monotonic behaviour was observed in the centreline viscosity, although we only show graphs corresponding to tubes of three different radii in Fig. 9. Referring to Fig. 10, the sharp decrease in $n$ and $N_{0} D e$ as one moves away from the axis of symmetry that is to be seen at higher Péclet numbers is replaced, when $P e=4.38 \times 10^{2}$, by an aggregate size distribution and associated viscosity that decay with $r$ much more gradually. The tube is now so small compared to a cell that, with the exception of the very near wall region, flow conditions are approximately uniform (plug-like). The non-monotonic progression of both $n$ and $N_{0} D e$ as functions of $r$ from the high to very low $P e$ regimes may be seen most clearly in Figs. 13 
and 14 , where the pressure gradient $P=-500 \mathrm{Nm}^{-3}$. At high values of $P e$ both variables show a steep decrease with $r$ and at the lower values of $P e, n$ and $N_{0} D e$ are more uniform in the bulk flow. Note the viscosity boundary layer, indicating the presence of a near-wall cell-depleted layer.

In summary, the model predicts, as it should, that blood becomes thinner as flow rates increase and that the viscosity assumes its maximum along the axis of symmetry, which is where both the greatest number density and the largest average size aggregates are to be found. The viscosity is a non-trivial function of both the local hematocrit (measured by $N_{0}$ ) and the average aggregate size (influenced strongly by the local shear stress). In the smallest vessels and at sufficiently high flow rates (see Fig. 10) the viscosity is, on the whole, higher in the bulk flow than in larger vessels but smaller, because of migration effects, in the near-wall region. Lower shear stresses in the smallest $P e$ flow means that the viscosity is larger and the velocity profile blunter near the centre. The thicker blood in the core more closely resembles a plug lubricated by plasma near the wall, whereas blood in the larger vessels is much more Newtonian in behaviour, with an almost parabolic velocity profile and very small elastic normal stresses. It may appear somewhat surprising, given the higher viscosity in the bulk flow at the lowest Péclet number compared to that in the flows at higher Péclet numbers, that the apparent viscosity should decrease as the tube radius decreases. This is due, however, to lubrication effects and (were the Fåhraeus effect to be taken account of) to a reduction in the tube hematocrit as the vessel diameter is made smaller.

We examine the phenomenon of decreasing apparent viscosity (the Fåhraeus-Lindqvist effect) and that of the decreasing tube hematocrit that accompanies it (the Fåhraeus effect) in the next section.

\subsection{Comparisons with in vitro experiments}

The conclusion of Barbee \& Cokelet (1971) that the Fåhraeus effect is entirely responsible for the Fåhraeus-Lindqvist effect has not been supported by subsequent experiments (Azelvandre \& Oiknine (1976)). In an effort to distinguish between the contributions of tube hematocrit and the presence of a wall slippage layer to the Fåhraeus-Lindqvist effect we consider three separate cases as the tube diameter (and, therefore, with it, the Péclet number $P e$ ) is reduced from $1 \mathrm{~mm}$ to $10 \mu \mathrm{m}$. These cases are as follows:

(a) Non-homogeneous model with tube hematocrit set equal to the feed/discharge hematocrit, 
(b) Non-homogeneous model with tube hematocrit determined experimentally by Pries et al. (1990),

(c) Homogeneous model (Fang \& Owens 2006; Owens 2006) with tube hematocrit determined experimentally by Pries et al. (1990).

In all three cases we will plot the predictions of the apparent viscosity relative to the plasma viscosity (a quantity which we denote by relative $\eta_{a p p}$ ) against tube diameter $D$, measured in $\mu m$. The feed/discharge hematocrit $H_{c}$ is held constant at 0.45 . Differences in our results between (a) and (b) above may be attributed to the Fåhraeus effect and those between (b) and (c) to the wall slip layer, since case (c) corresponds to the limit $D \rightarrow \infty$ and uniform cell number density. Comparison is made with the empirical curve of Pries et al. (1992)

$$
\text { relative } \eta_{a p p, 0.45}=220 \exp (-1.3 D)+3.2-2.44 \exp \left(-0.06 D^{0.645}\right)
$$

(with $D$ measured in $\mu \mathrm{m}$ ), the parameters in which were determined by fitting the curve to 163 data points coming from 18 experimental studies performed over a period of some 60 years. The measurements in the studies were made at feed hematocrits of between 0.4 and 0.45 and where $H_{c}$ was below 0.45 an extrapolated relative $\eta_{a p p}$ was calculated. Even allowing for the fact that apparent viscosities were calculated relative to the suspending medium used (which was not always plasma) it is remarkable (see Fig. 2. of Pries et al. (1992)), given the very different conditions under which the experiments were performed (anticoagulant, tube length, temperature, pressure drop, method of determination of the apparent viscosity and even animal species) that scatter of the experimental data points about the curve (3.4) is not greater than it appears to be. The relative apparent viscosity tended consistently to a value of approximately 3.2 in tubes of diameter $1 \mathrm{~mm}$ but this does not appear to us to be the asymptotic value from the data of Fig. 2. of Pries et al. (1992) (which is still increasing with $D$ ) and this motivated the choice of $\eta_{\infty}=0.00234 P$ a.s made in our model, thus yielding a relative $\eta_{a p p} \approx 3.34$ as $D \rightarrow \infty$. The pressure gradient $P$ for all results presented in this section was fixed at $-1000 \mathrm{Nm}^{-3}$.

\subsubsection{The Fåhraeus effect}

At this stage in the development of the non-homogeneous model proposed in this paper, no satisfactory equation predicting the tube hematocrit $H_{c t}$ from the flow conditions and material 
parameters is available. However, using the empirical parametric description of the Fåhraeus effect given by Pries et al. (1990):

$$
\frac{H_{c t}}{H_{c}}=H_{c}+\left(1-H_{c}\right)(1+1.7 \exp (-0.35 D)-0.6 \exp (-0.01 D)),
$$

(with $D$ measured in $\mu m$ ) we are able to fit the tube hematocrit by adjusting $\eta_{\infty}$. This is done by returning to the definition $(2.26)$ of $\eta_{\infty}$ :

$$
\eta_{\infty}:=N_{a v}\left(k_{B} T+\kappa\right) \lambda_{H}
$$

and then using (2.19)-(2.20) to conclude that

$$
H_{c t}=\frac{\eta_{\infty} V_{e}}{\left(k_{B} T+\kappa\right) \lambda_{H}} \Rightarrow \eta_{\infty}(D)=\frac{H_{c t}(D)}{H_{c t}(\infty)} \eta_{\infty}(\infty),
$$

where, as before, $V_{e}$ is the volume of a single erythrocyte. The fitted curve for $H_{c t}$ as determined from (3.6) and the empirical (3.5) is shown in Fig. 15. Of course, the fitting does not rely in any way upon the model being non-homogeneous and exactly the same procedure may be followed for the homogeneous model of Fang \& Owens (2006) and Owens (2006). The upturn in $H_{c t}$ for $D$ sufficiently small and referred to earlier in our discussion, is to be seen for $D \lesssim 15 \mu m$. The corresponding upturn in the apparent viscosity occurs at a yet smaller $D$, however.

\subsubsection{The Fåhraeus-Lindqvist effect}

In Fig. 16 we show the relative apparent viscosity as a function of tube diameter predicted by the non-homogeneous model both when the tube hematocrit is fitted from (3.6) and when the tube hematocrit is fixed equal to its feed/discharge value of 0.45 . Although agreement with the experimental data in the case of a constant tube hematocrit (fixed $\eta_{\infty}$ ) is good it is significantly better, and especially so at intermediate values of $D$, when a realistic $H_{c t}$ is used. From the results shown in Fig. 16 it would not seem that the apparent relative viscosity is a sensitive function of tube hematocrit, although tube hematocrit clearly plays a non-negligible role in determining the apparent viscosity. This is in contradiction, therefore, to the contention by Barbee \& Cokelet (1971) that the Fåhraeus effect is completely responsible for the Fåhraeus-Lindqvist effect.

When the homogeneous model with fitted $H_{c t}$ is used in the same experiment the upper curve of Fig. 17 results. To be absolutely sure that this is correctly calculated we have used separate codes employing both the finite element method described in Section 3.2 and the finite difference code 
of Fang \& Owens (2006) with no-slip boundary conditions. Differences in the homogeneous model plot and the non-homogeneous model plot may now be attributed to diffusion (migration) effects and the consequent appearance of a cell-depleted wall slip layer, since in the homogeneous model the number density of cells is always a constant across the tube radius. The dramatic rise in the relative apparent viscosity as $D \rightarrow 0$ manifested in the homogeneous model curve is reminiscent of that observed experimentally, for example, in the case of heated red blood cells or those fixed with glutaraldehyde, where in the former case the cells are less deformable than normal cells and in the latter are rigid (McKay \& Meiselman (1988)). Changing the deformability of red cells in this way changes the migration properties of the same, and was shown by McKay \& Meiselman (1988) to result in reversal of the Fåhraeus-Lindqvist effect in tubes of diameters below approximately $150 \mu \mathrm{m}$, even though the tube hematocrit of the heated red blood cells continued to decrease with tube diameter beyond this point. In the homogeneous model, the asymptotic value of the relative $\eta_{\text {app }}$ as $D \rightarrow 0$ is that corresponding to the total zero shear rate viscosity $\left(\eta_{0}+\eta_{N}\right) / \eta_{N}$. This is due to the fact that the polymeric centreline viscosity is always $\eta_{0}$, the number density $N_{0}$ being constant everywhere and the average aggregate size $n$ in steady flow being a function only of the local shear rate.

\section{Conclusions}

In this paper we have presented a non-homogeneous model for whole human blood, taking account of fragmentation and aggregation of the aggregates and stress-induced diffusion. The magnitude of the elastic normal stress component driving particle migration in small tubes has been shown to depend on cell deformability. Predictions of the Fåhraeus-Lindqvist effect in narrow tubes match closely the experimental data and the relative importance of the Fåhraeus effect and of a cell depleted layer near the wall on the apparent viscosity has been discussed. We conclude that the Fåhraeus effect is influential but not determinative in this regard. 


\section{Appendix A. Definition of aggregation and disaggregation functions and parameter values.}

Motivated by the work of Murata \& Secomb (1988) and of Shiga et al. (1983) the aggregation rate $a(\dot{\gamma}) N_{a v, h o m}$ is defined as:

$$
a(\dot{\gamma}) N_{a v, h o m}=\left\{\begin{array}{cl}
a_{1,3} \dot{\gamma}^{3}+a_{1,2} \dot{\gamma}^{2}+a_{1,0} & \text { for } 0 \leq \dot{\gamma} \leq \dot{\gamma}_{c}, \\
a_{2,3} \dot{\gamma}^{3}+a_{2,2} \dot{\gamma}^{2}+a_{2,1} \dot{\gamma}+a_{2,0} & \text { for } \dot{\gamma}_{c} \leq \dot{\gamma} \leq \dot{\gamma}_{\max } \\
0 & \text { for } \dot{\gamma}>\dot{\gamma}_{\max }
\end{array}\right.
$$

where

$$
\begin{gathered}
a_{1,0}=1, \\
a_{1,2}=3 a_{1,0} / \dot{\gamma}_{c}^{2}, \\
a_{1,3}=-2 a_{1,0} / \dot{\gamma}_{c}^{3}, \\
a_{2,0}=\frac{2 a_{1,0} \dot{\gamma}_{\max }^{2}\left(-\dot{\gamma}_{\max }+3 \dot{\gamma}_{c}\right)}{-\dot{\gamma}_{\max }^{3}-3 \dot{\gamma}_{\max } \dot{\gamma}_{c}^{2}+\dot{\gamma}_{c}^{3}+3 \dot{\gamma}_{c} \dot{\gamma}_{\max }^{2}} \\
a_{2,1}=\frac{-12 \dot{\gamma}_{c} a_{1,0} \dot{\gamma}_{\max }}{-\dot{\gamma}_{\max }^{3}-3 \dot{\gamma}_{\max } \dot{\gamma}_{c}^{2}+\dot{\gamma}_{c}^{3}+3 \dot{\gamma}_{c} \dot{\gamma}_{\max }^{2}} \\
a_{2,2}=\frac{6 a_{1,0}\left(\dot{\gamma}_{\max }+\dot{\gamma}_{c}\right)}{-\dot{\gamma}_{\max }^{3}-3 \dot{\gamma}_{\max } \dot{\gamma}_{c}^{2}+\dot{\gamma}_{c}^{3}+3 \dot{\gamma}_{c} \dot{\gamma}_{\max }^{2}}, \\
a_{2,3}=\frac{-4 a_{1,0}}{-\dot{\gamma}_{\max }^{3}-3 \dot{\gamma}_{\max } \dot{\gamma}_{c}^{2}+\dot{\gamma}_{c}^{3}+3 \dot{\gamma}_{c} \dot{\gamma}_{\max }^{2}},
\end{gathered}
$$

and where the critical shear rate (see Murata \& Secomb (1988)) is chosen as $\dot{\gamma}_{c}=5.78 \mathrm{~s}^{-1}$ and the maximum shear rate $\dot{\gamma}_{\max }=900 \mathrm{~s}^{-1} . N_{a v, h o m}$ is the infinite radius tube average cell number density, defined (cf. Eqn. (2.20)) as

$$
N_{a v, h o m}=\frac{H_{c t}(\infty)}{V_{e}}=\frac{H_{c}}{V_{e}} .
$$

Therefore, $N_{a v, h o m} / N_{a v}=H_{c} / H_{c t}$.

$b(\dot{\gamma})$ is determined from $a(\dot{\gamma}) N_{a v}$ from the relationship that holds in steady homogeneous flow (see Eqns (27) and (28) of Owens (2006)):

$$
b(\dot{\gamma})=\frac{a(\dot{\gamma}) N_{a v, h o m}}{n_{s t}\left(n_{s t}-1\right)},
$$

where

$$
n_{s t}:=\frac{\eta_{s t}(\dot{\gamma})}{\eta_{\infty}}\left(1+\frac{3}{2} a(\dot{\gamma}) N_{a v, h o m} \lambda_{H}\right)
$$




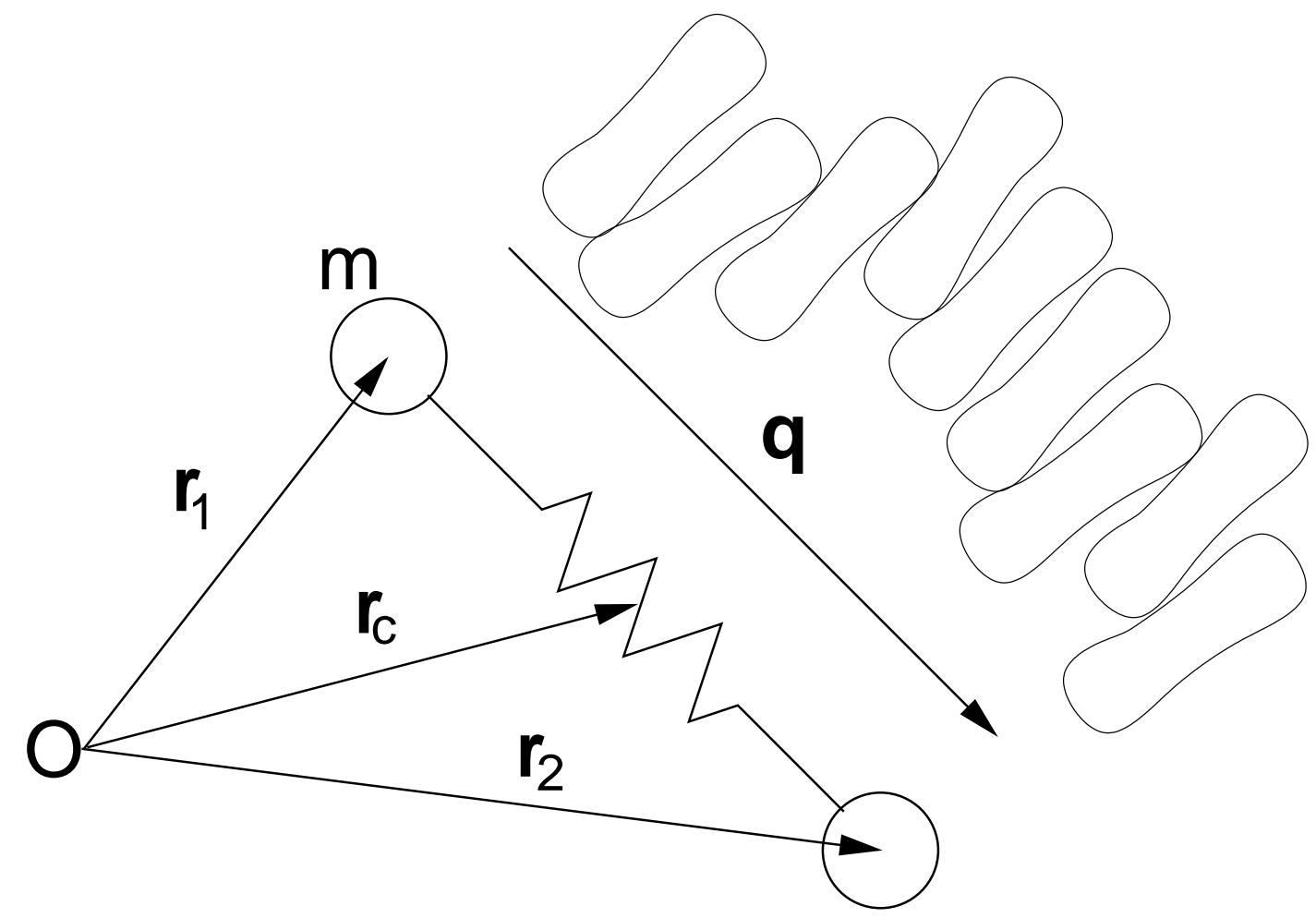

$\mathrm{m}$

Figure 1. Single Hookean dumbbell and its representation of the end-to-end vector of a simple rouleau. A linear spring connects two point masses $m$ having position vectors $\boldsymbol{r}_{1}$ and $\boldsymbol{r}_{2}$. The end-to-end vector $\boldsymbol{q}=\boldsymbol{r}_{2}-\boldsymbol{r}_{1}$ and the position vector of the centre of mass $\boldsymbol{r}_{c}=\left(\boldsymbol{r}_{1}+\boldsymbol{r}_{2}\right) / 2$.

and

$$
\eta_{s t}(\dot{\gamma})=\eta_{0}\left(\frac{1+\theta \dot{\gamma}^{m}}{1+\beta \dot{\gamma}^{m}}\right),
$$

with $\theta / \beta:=\eta_{\infty} / \eta_{0}$, is the steady polymeric viscosity of Cross type (Cross (1965)), fitted to steady shear data. 
(a)

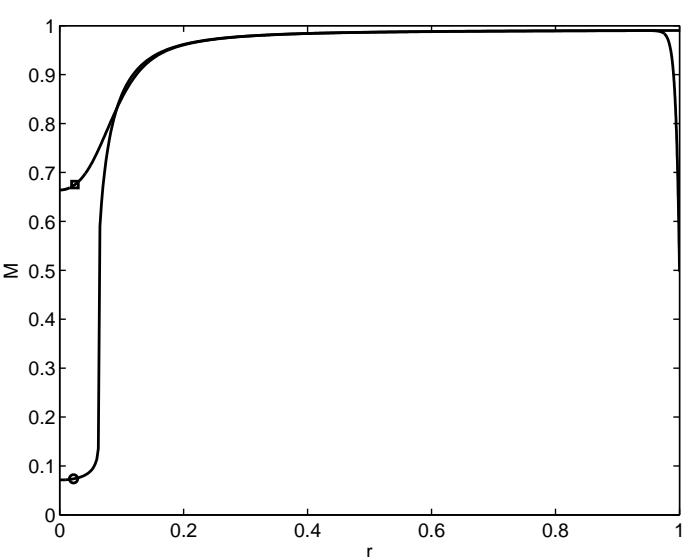

(b) $\begin{array}{rrrrrrrrr}0.4 & 1 & 1 & 1 & 1 & 1 & 1 & 1 & 1 \\ 0.965 & 0.97 & 0.975 & 0.98 & 0.985 & 0.99 & 0.995 & 1 & 1.005\end{array}$

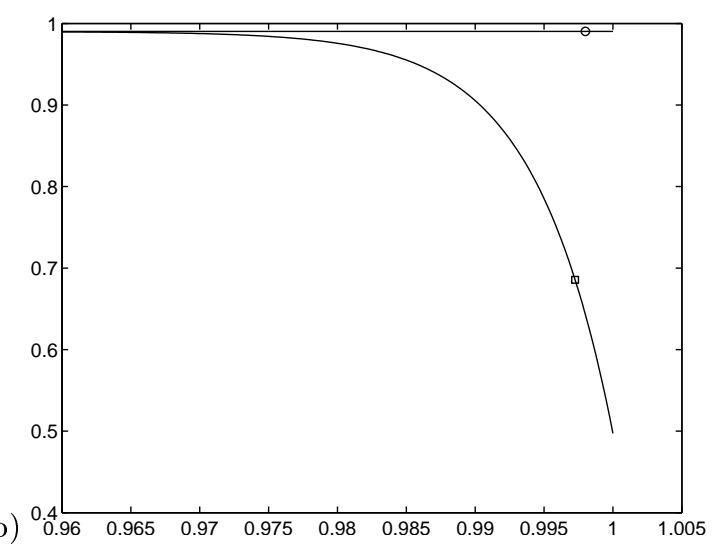

Figure 2. (a) Comparison of aggregate number density $M$ for $\circ$ : homogeneous and $\square$ : non-homogeneous flow, against normalized radial distance $r$. $P=-1000 \mathrm{Nm}^{-3}, R=700 \mu \mathrm{m}, P e=85750$. (b) Zoom of the boundary layer near $r=1$.
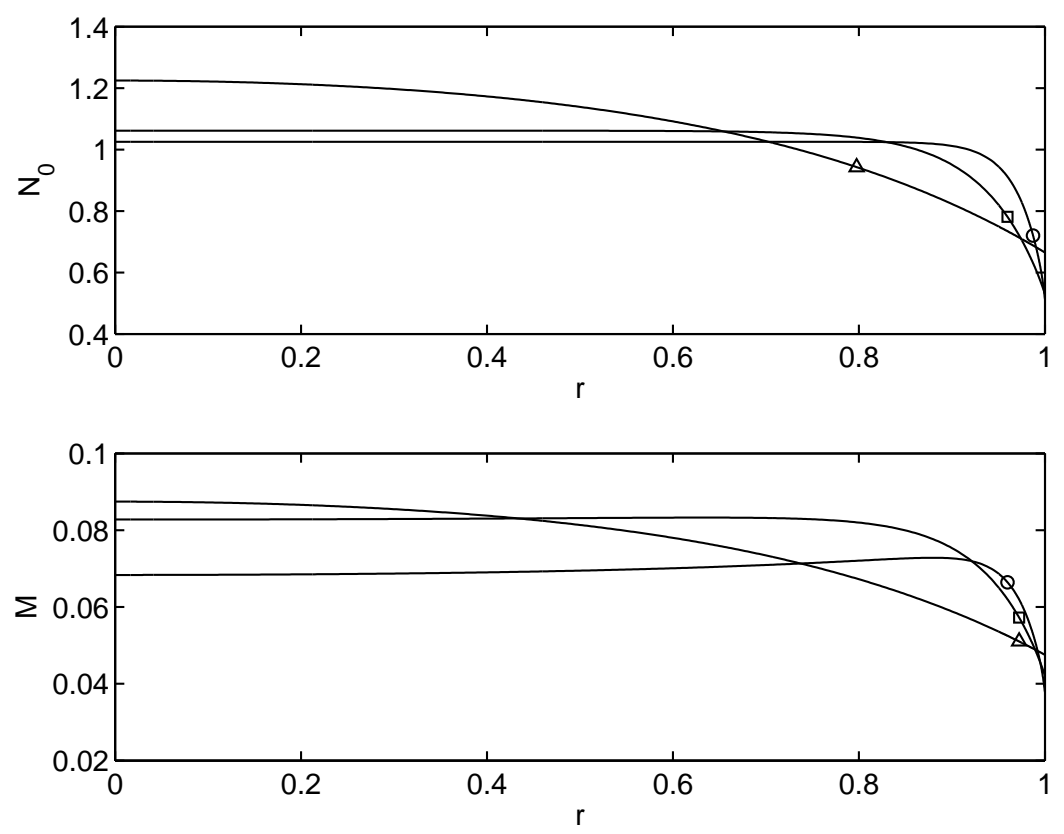

Figure 3. Cell number density $N_{0}$ and aggregate number density $M$ against normalized radial distance r. $P=-10 \mathrm{Nm}^{-3}$ ○: $R=500 \mu m, P e=4.38 \times 10^{4}, \square: R=250 \mu m, P e=1.09 \times 10^{4}, \Delta: R=50 \mu m$, $P e=4.38 \times 10^{2}$.

\section{REFERENCES}

Azelvandre, F. \& Oiknine, C. 1976 Effet Fahraeus et effet Fahraeus-Lindqvist: résultats expérimentaux et modèles théoriques. Biorheology 13, 325-335.

BAGChi, P. 2007 Mesoscale simulation of blood flow in small vessels. Biophys. J. 92, 1858-1877.

Barbee, J. H. \& Cokelet, G. R. 1971 Predictions of blood flow in tubes with diameters as small as $29 \mu \mathrm{m}$. Microvascular Research 3, 17-21. 

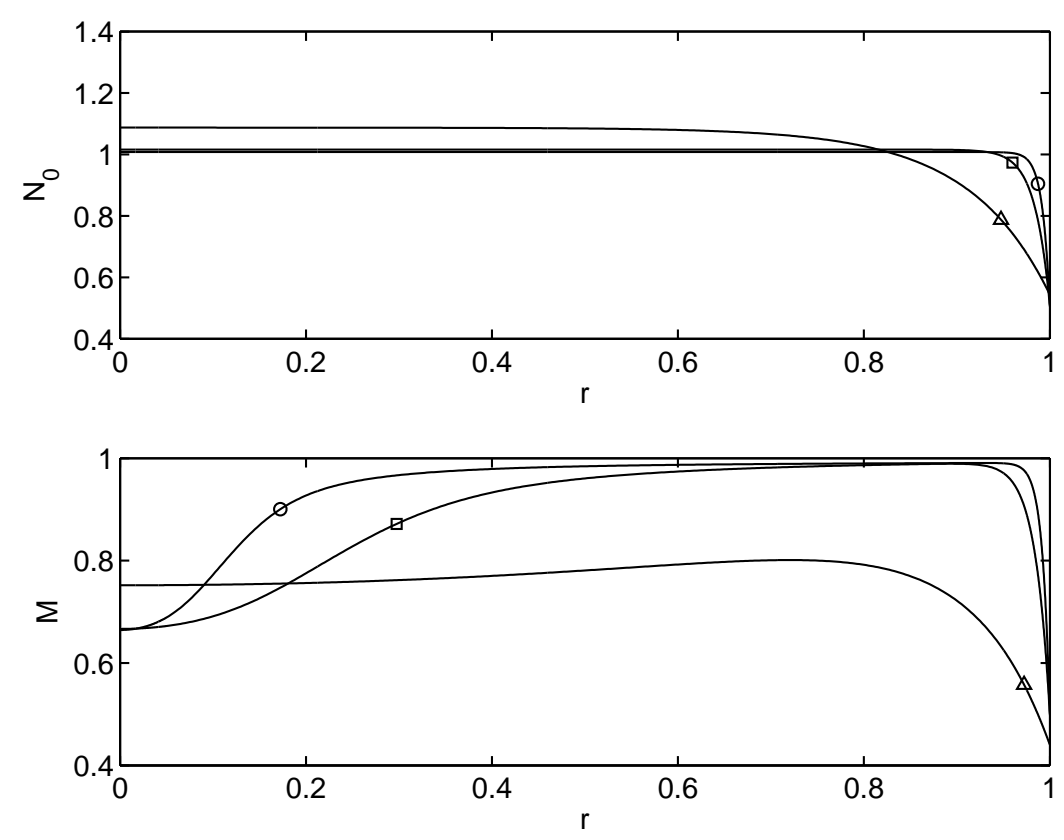

Figure 4. Cell number density $N_{0}$ and aggregate number density $M$ against normalized radial distance r. $P=-1000 \mathrm{Nm}^{-3}$ ○: $R=500 \mu m, P e=4.38 \times 10^{4}, \square: R=250 \mu m, P e=1.09 \times 10^{4}, \Delta: R=50 \mu m$, $P e=4.38 \times 10^{2}$.
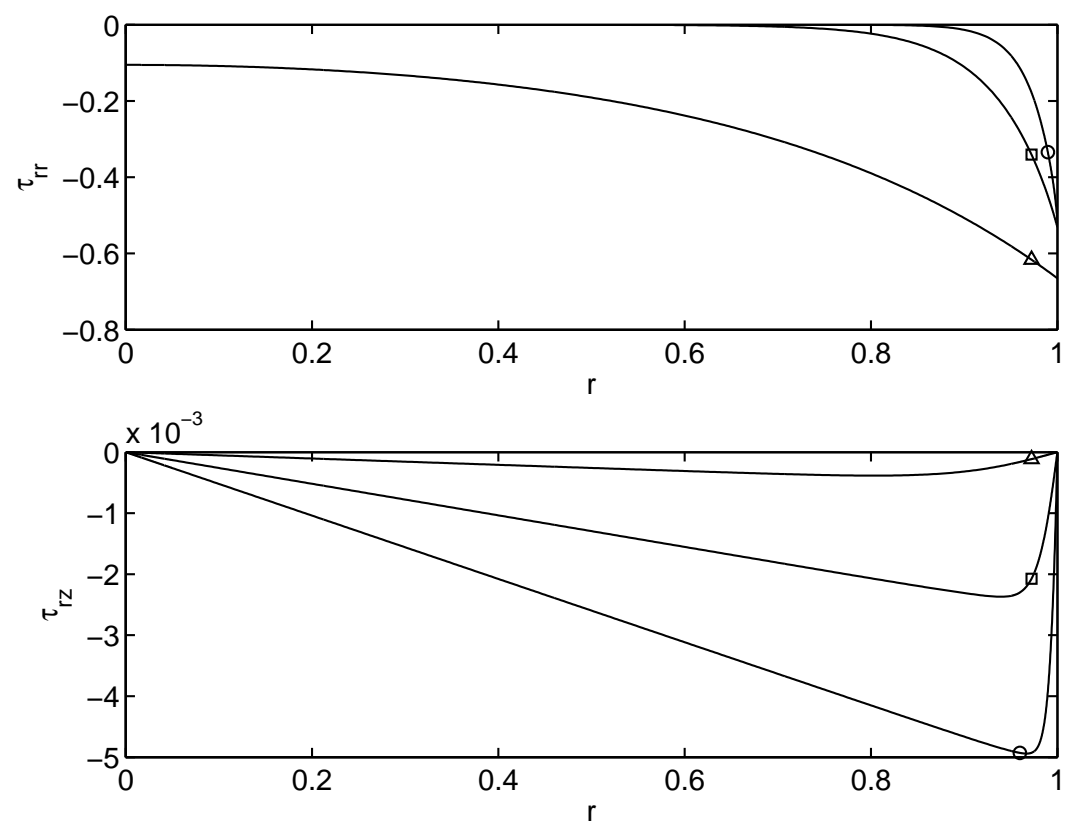

Figure 5. Radial elastic normal stress $\tau_{r r}$ and elastic shear stress $\tau_{r z}$ against normalized radial distance r. $P=-10 N m^{-3}$ ○: $R=500 \mu m, P e=4.38 \times 10^{4}, \square: R=250 \mu m, P e=1.09 \times 10^{4}, \Delta: R=50 \mu m$, $P e=4.38 \times 10^{2}$. 

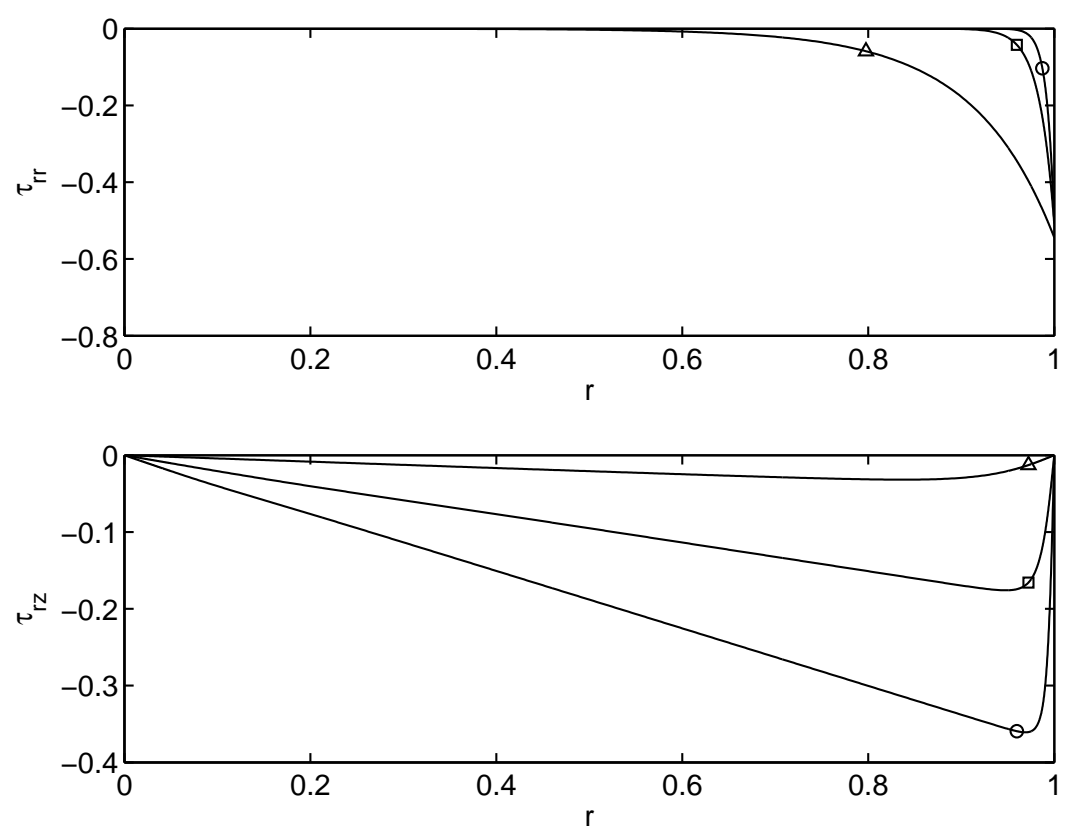

FigURE 6. Radial elastic normal stress $\tau_{r r}$ and elastic shear stress $\tau_{r z}$ against normalized radial distance r. $P=-1000 \mathrm{Nm}^{-3}$ o: $R=500 \mu m, P e=4.38 \times 10^{4}, \square: R=250 \mu m, P e=1.09 \times 10^{4}, \Delta: R=50 \mu m$, $P e=4.38 \times 10^{2}$.
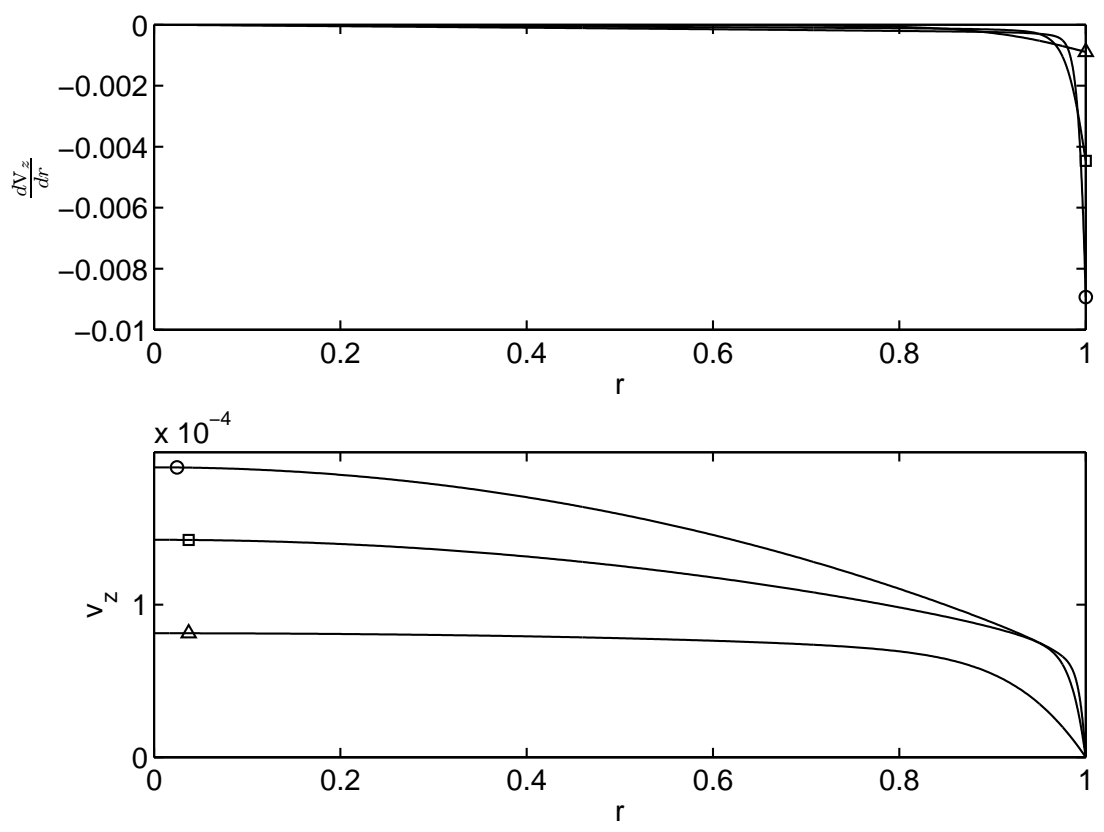

Figure 7. Axial velocity gradient $d v_{z} / d r$ and axial velocity $v_{z}$ against normalized radial distance $r$. $P=-10 \mathrm{Nm}^{-3}$ ○: $R=500 \mu m, P e=4.38 \times 10^{4}, \square: R=250 \mu m, P e=1.09 \times 10^{4}, \Delta: R=50 \mu m$, $P e=4.38 \times 10^{2}$. 

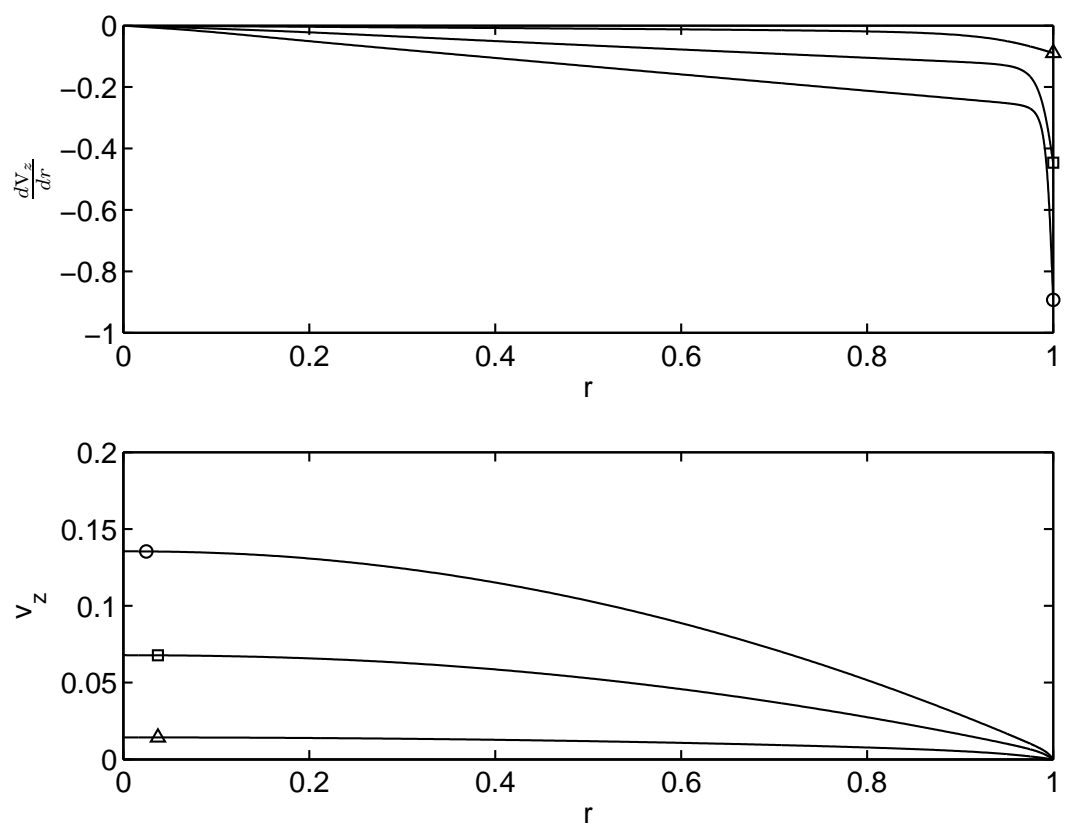

Figure 8. Axial velocity gradient $d v_{z} / d r$ and axial velocity $v_{z}$ against normalized radial distance $r$. $P=-1000 \mathrm{Nm}^{-3} \circ: R=500 \mu \mathrm{m}, \mathrm{Pe}=4.38 \times 10^{4}, \square: R=250 \mu \mathrm{m}, P e=1.09 \times 10^{4}, \Delta: R=50 \mu m$, $P e=4.38 \times 10^{2}$.
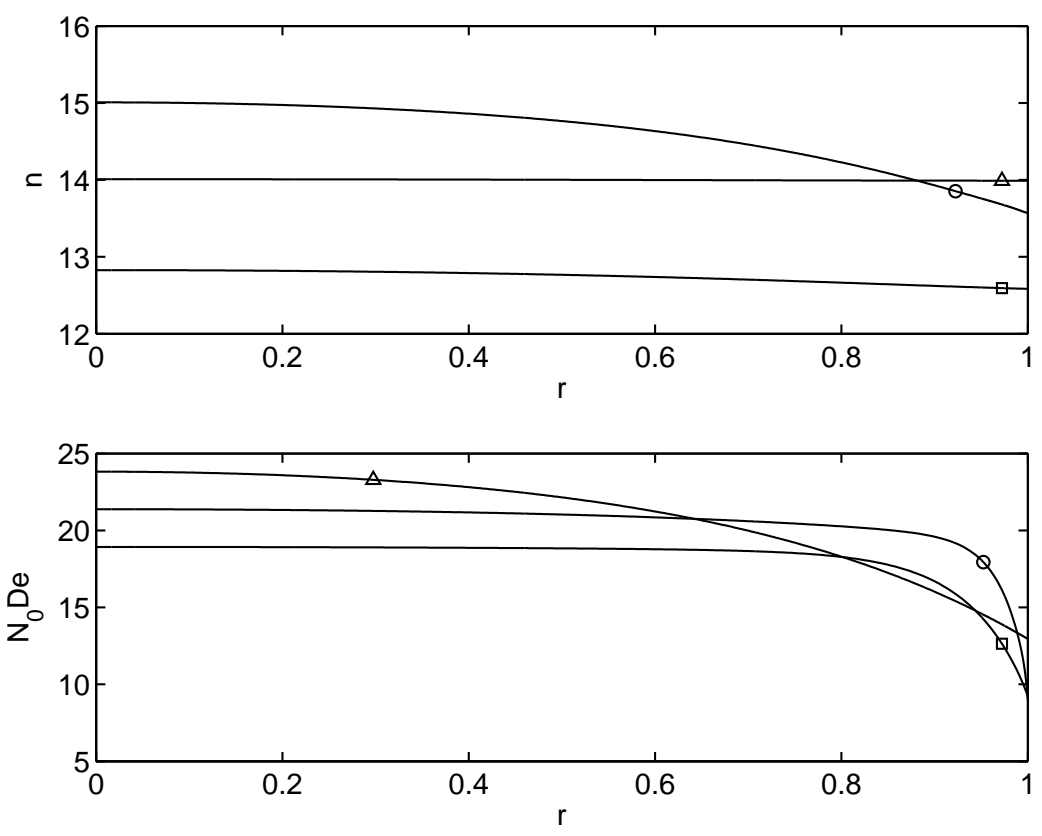

Figure 9. Average aggregate size $n$ and viscosity $N_{0} D e$ against normalized radial distance $r$. $P=-10 \mathrm{Nm}^{-3} \circ: R=500 \mu \mathrm{m}, P e=4.38 \times 10^{4}, \square: R=250 \mu m, P e=1.09 \times 10^{4}, \triangle: R=50 \mu m$, $P e=4.38 \times 10^{2}$. 

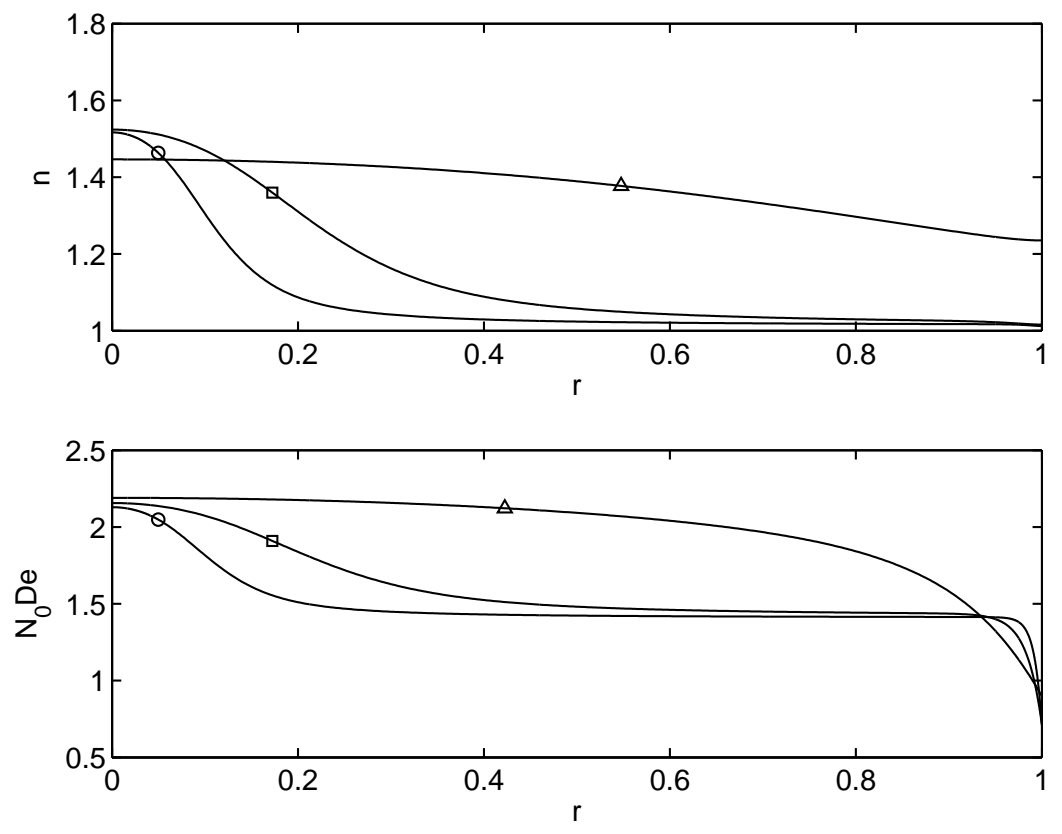

FiguRE 10. Average aggregate size $n$ and viscosity $N_{0} D e$ against normalized radial distance $r$.

$$
\begin{gathered}
P=-1000 N m^{-3} \circ: R=500 \mu m, P e=4.38 \times 10^{4}, \square: R=250 \mu m, P e=1.09 \times 10^{4}, \triangle: R=50 \mu m, \\
P e=4.38 \times 10^{2} .
\end{gathered}
$$

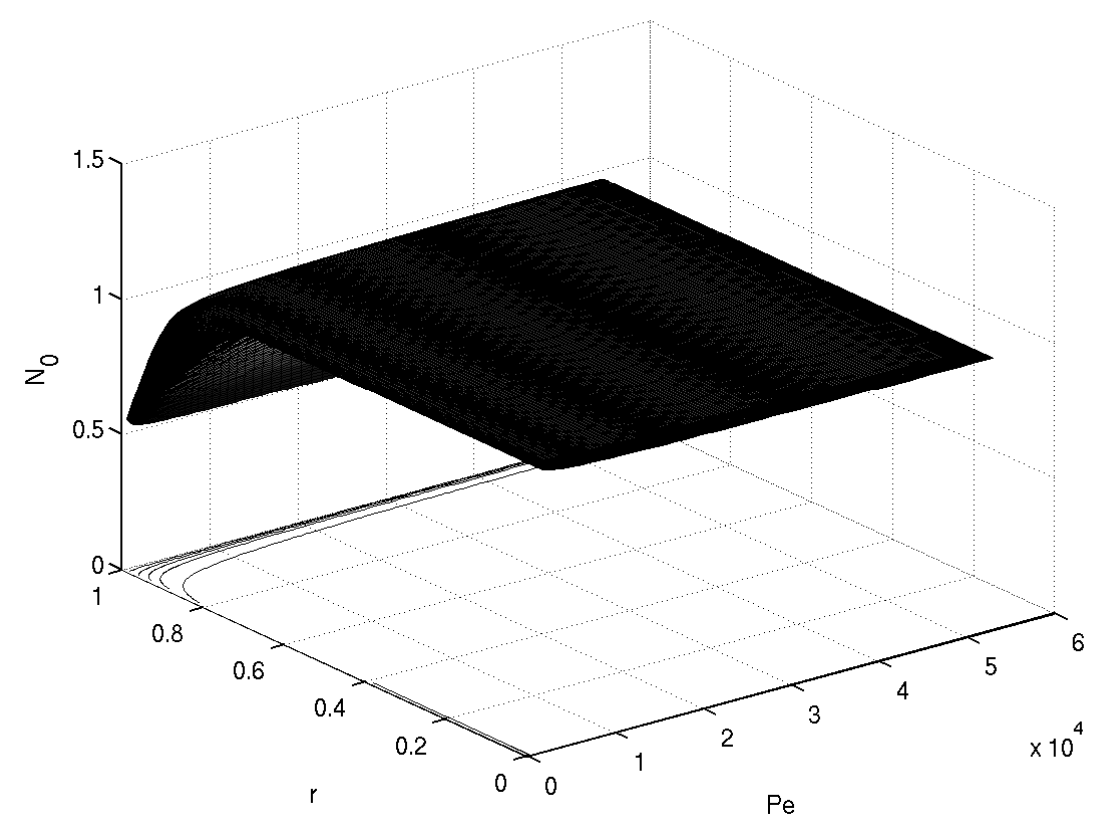

Figure 11. Cell number density $N_{0}$ as a function of normalized radial distance $r$ and Péclet number Pe.

$$
P=-500 \mathrm{Nm}^{-3} \text {. }
$$

Baskurt, O. K. \& Meiselman, H. J. 2003 Blood rheology and hemodynamics. Semin. Thromb. Hemost. 29, 435-450.

Beris, A. N. \& Mavrantzas, V. G. 1994 On the compatibility between various macroscopic formalisms for the concentration and flow of dilute polymer solutions. J. Rheol. 38, 1235-1250. 


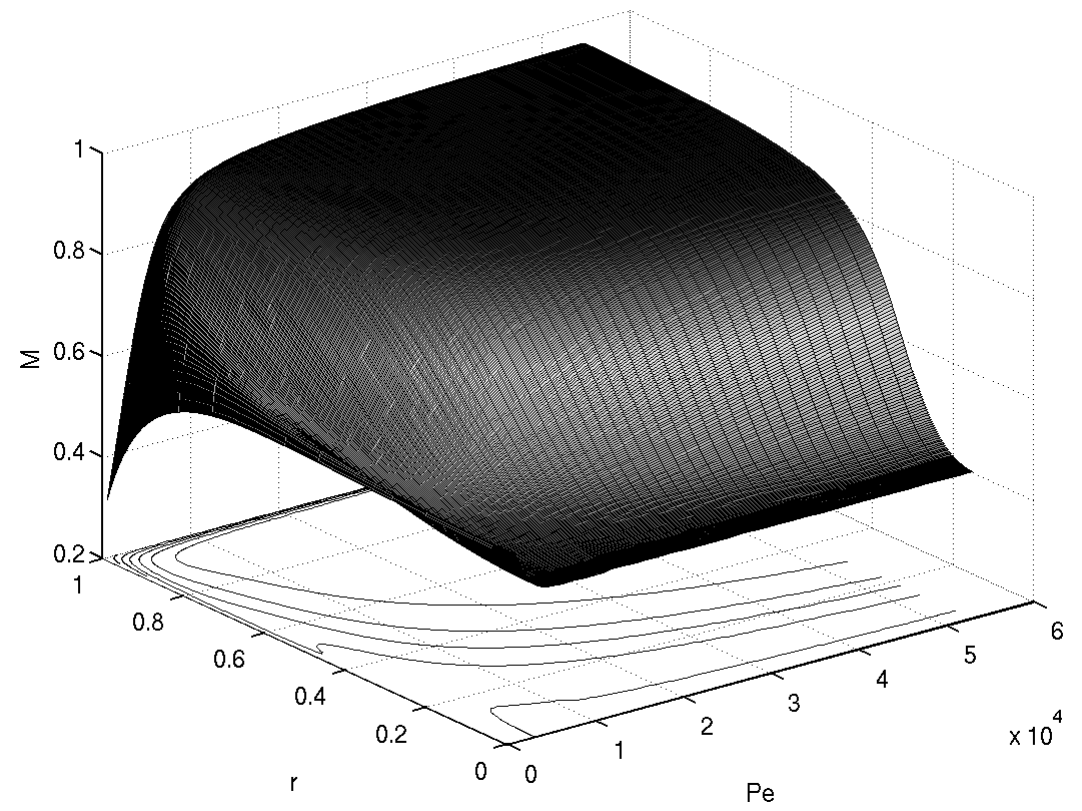

Figure 12. Aggregate number density $M$ as a function of normalized radial distance $r$ and Péclet number Pe. $P=-500 \mathrm{Nm}^{-3}$.

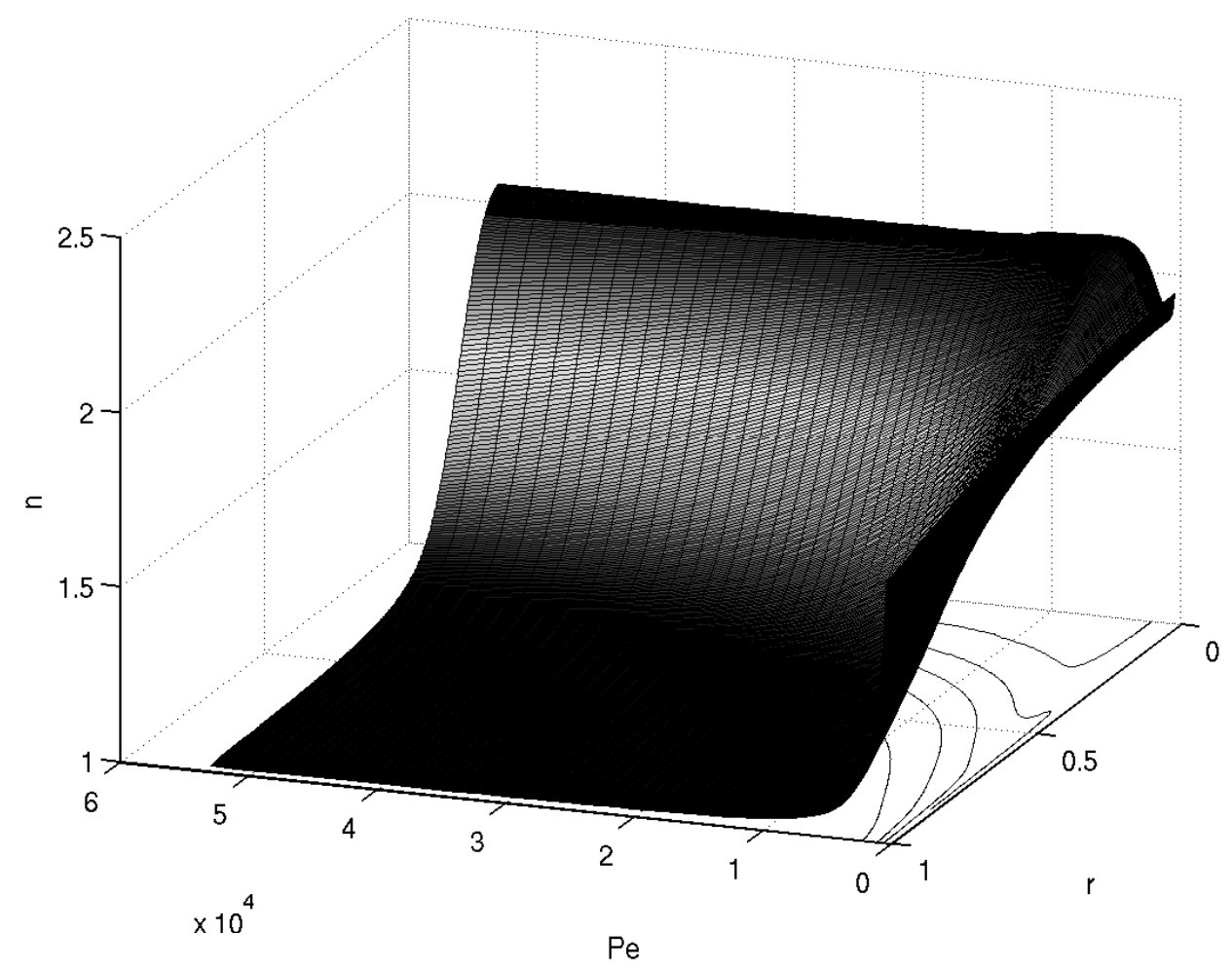

Figure 13. Average aggregate size $n$ as a function of normalized radial distance $r$ and Péclet number $P e$.

$$
P=-500 \mathrm{Nm}^{-3} \text {. }
$$




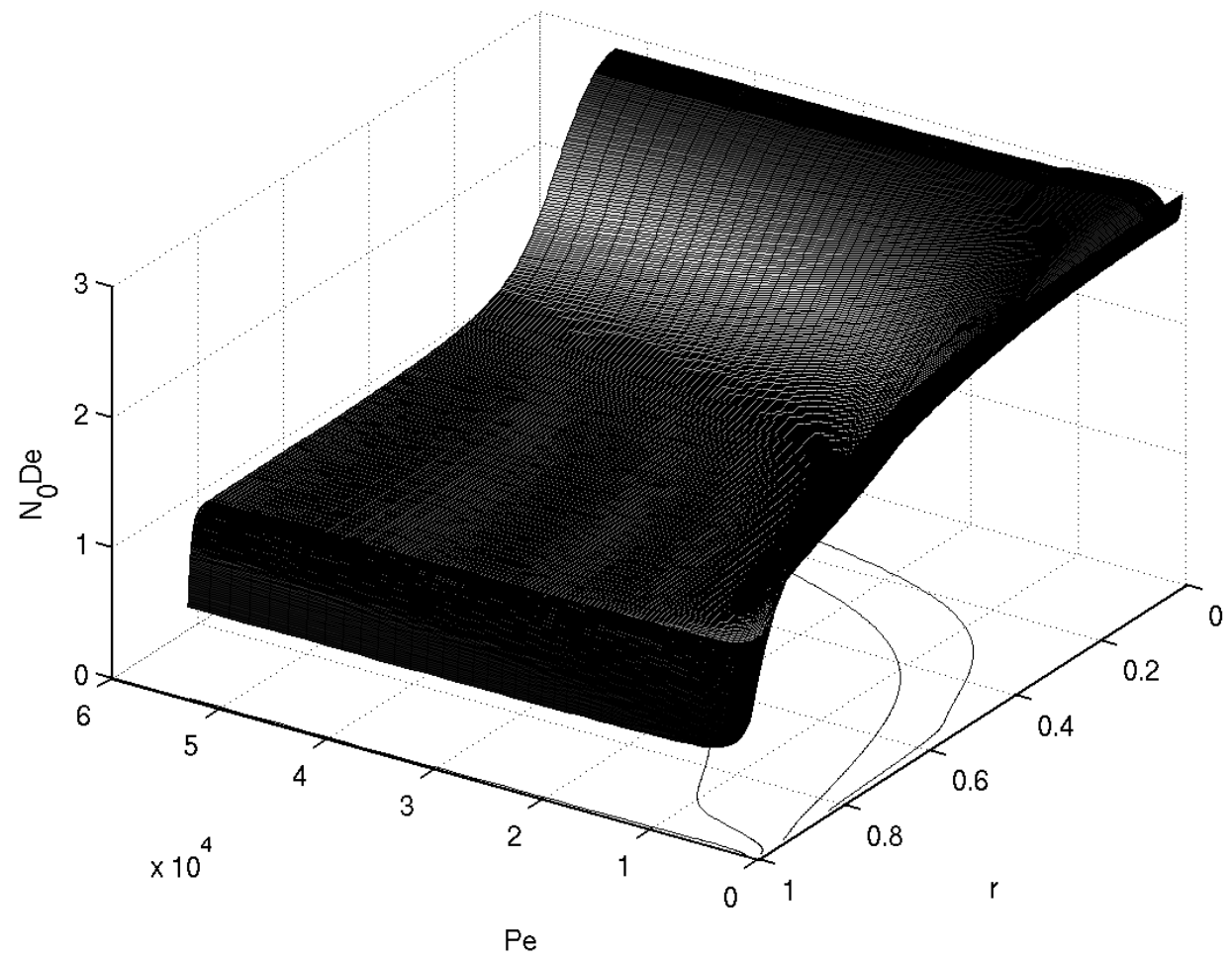

Figure 14. Viscosity $N_{0} D e$ as a function of normalized radial distance $r$ and Péclet number Pe.

$$
P=-500 \mathrm{Nm}^{-3} \text {. }
$$

Bhave, A. V., Armstrong, R. C. \& Brown, R. A. 1991 Kinetic theory and rheology of dilute, nonhomogeneous polymer solutions. J. Chem. Phys. 95, 2988-3000.

Blair, G. W. Scott 1958 The importance of the sigma phenomenon in the study of the flow of blood. Rheol. Acta 1, 123-126.

Bugliarello, G. \& Sevilla, J. 1970 Velocity distribution and other characteristics of steady and pulsatile blood flow in fine glass tubes. Biorheology 7, 85-107.

Bureau, M., Healy, J. C., Bourgoin, D. \& Joly, M. 1980 Rheological hysteresis of blood at low shear rate. Biorheology 17, 191-203.

Caro, C. G., Pedley, T. J., Schroter, R. C. \& Seed, W. A. 1978 The Mechanics of the Circulation. Oxford University Press.

Chien, S. \& JAn, K.-M. 1973 Ultrastructural basis of the mechanism of rouleaux formation. Microvascular Res. 5, 155-166.

Cook, L. P. \& Rossi, L. F. 2004 Slippage and migration in models of dilute wormlike micellar solutions and polymeric fluids. J. Non-Newtonian Fluid Mech. 116, 347-369.

Cross, M. M. 1965 Rheology of non-Newtonian fluids: a new flow equation for pseudoplastic systems. $J$. Colloid Sci. 20, 417-437.

FAnraeus, R. 1929 The suspension stability of the blood. Physiol. Rev. 9, 241-274. 


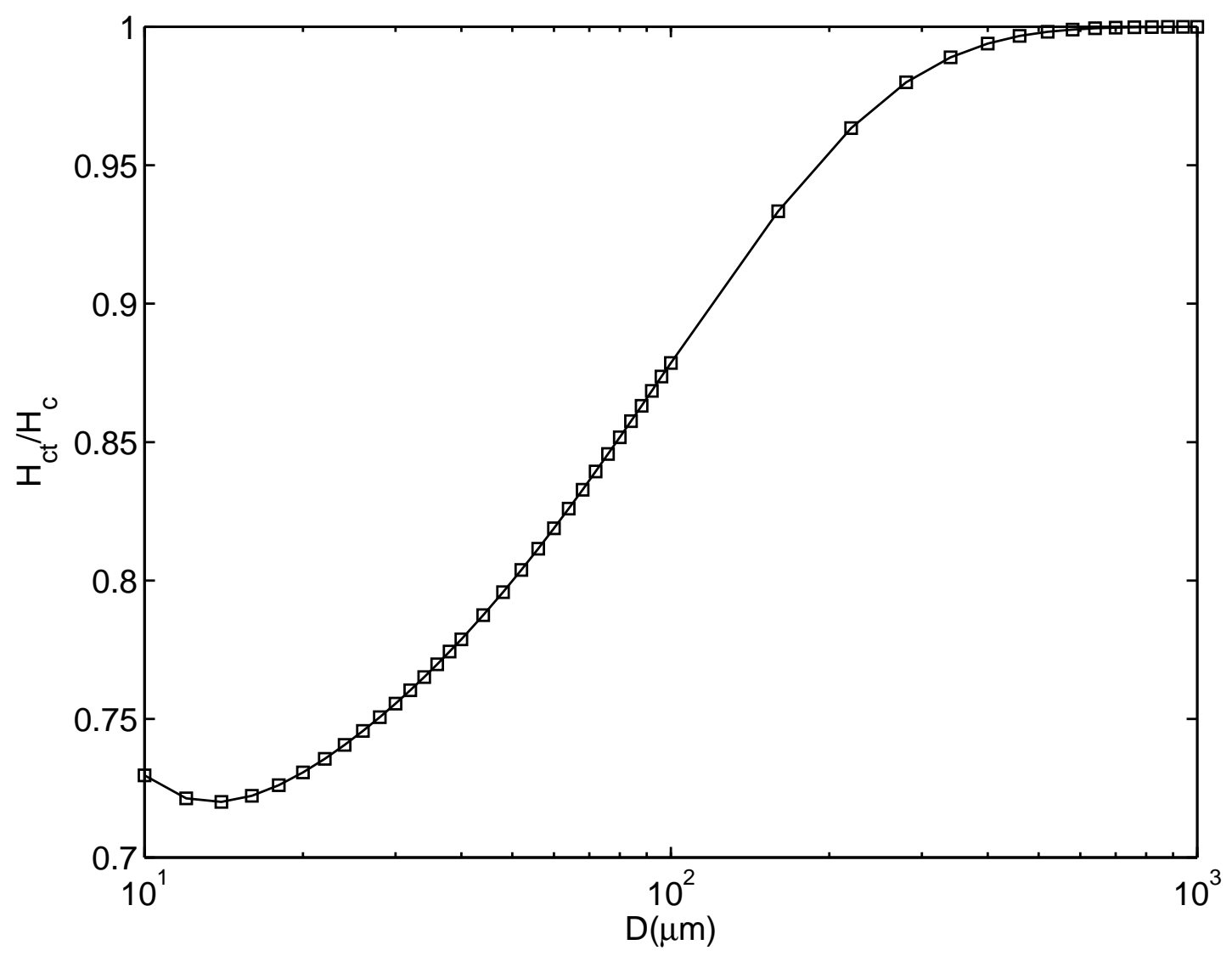

Figure 15. Fåhraeus effect. Relative tube hematocrit $H_{c t} / H_{c}$ against tube diameter $D$ $(\mu m) . \quad P=-1000 \mathrm{Nm}^{-3}$. Fit of non-homogeneous model $(\square)$ to the empirical formula $H_{c t} / H_{c}=H_{c}+\left(1-H_{c}\right)(1+1.7 \exp (-0.35 D)-0.6 \exp (-0.01 D))($ Pries et al. $(1990))$.

FÅnraeus, R. \& Lindqvist, T. 1931 The viscosity of the blood in narrow capillary tubes. Am. J. Physiol. 96, $562-568$.

FAng, J. \& Owens, R. G. 2006 Numerical simulations of pulsatile blood flow using a new constitutive model. Biorheology 43, 637-660.

Gaehtgens, P., Albrecht, K. H. \& Kreutz, F. 1978 Fahraeus effect and cell screening during tube flow of human blood. I. Effect of variation of flow rate. Biorheology 15, 147-154.

Goldsmith, H. L., Cokelet, G. R. \& Gaentgens, P. 1989 Robin Fåhraeus: evolution of his concepts in cardiovascular physiology. Am. J. Physiol. 257 (Heart Circ. Physiol. 26), H1005-H1015.

Goldsmith, H. L. \& Marlow, J. C. 1979 Flow behavior of erythrocytes. II. Particle motions in concentrated suspensions of ghost cells. J. Colloid Interface Sci. 71, 383-407.

Kramers, H. A. 1944 Het gedrag van macromoleculen in een stroomende vloeistof. Physica 11, 1-19.

Liu, Y. \& Liu, W. K. 2006 Rheology of red blood cell aggregation by computer simulation. J. Comp. Phys. 220, 139-154.

Mayer, G. 1965 Anomalous viscosity of human blood. Am. J. Physiol. 208, 1267-1269. 


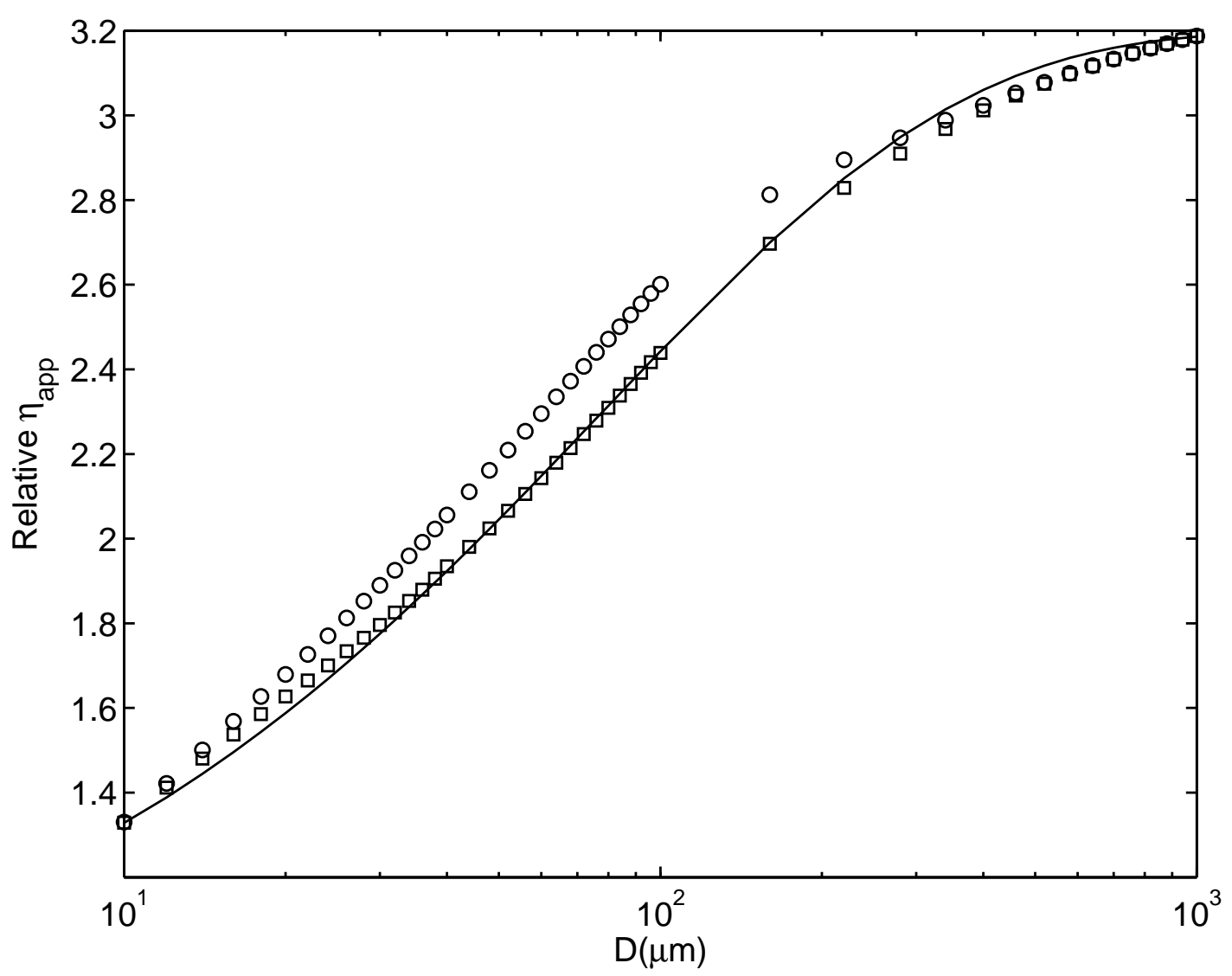

Figure 16. Fåhraeus-Lindqvist effect. Apparent relative viscosity $\eta_{a p p}$ against tube diameter $D(\mu m)$. $P=-1000 \mathrm{Nm}^{-3}$. — : $\eta_{\text {app }}=220 \exp (-1.3 D)+3.2-2.44 \exp \left(-0.06 D^{0.645}\right)$ (Pries et al. (1992)). ○: non-homogeneous model with constant tube hematocrit $H_{c t}=H_{c}$. $\square$ : non-homogeneous model with $H_{c t} / H_{c}=H_{c}+\left(1-H_{c}\right)(1+1.7 \exp (-0.35 D)-0.6 \exp (-0.01 D))($ Pries et al. $(1990))$.

McKay, C. B. \& Meiselman, H. J. 1988 Osmolality-mediated Fahraeus and Fahraeus-Lindqvist effects for human RBC suspensions. Am. J. Physiol. 254 (Heart Circ. Physiol. 23), H238-H249.

Moyers-Gonzalez, M. \& Owens, R. G. 2008 A non-homogeneous constitutive model for human blood. Part II: Asymptotic solution for large Péclet numbers. J. Non-Newtonian Fluid Mech. .

Moyers-Gonzalez, M., Owens, R. G. \& Fang, J. 2008 A non-homogeneous constitutive model for human blood. Part III: Oscillatory flow. J. Non-Newtonian Fluid Mech. .

Murata, T. \& Secomb, T. W. 1988 Effects of shear rate on rouleau formation in simple shear flow. Biorheology 25, 113-122.

Onuki, A. 2002 Phase Transition Dynamics. Cambridge University Press.

Owens, R. G. 2006 A new microstructure-based constitutive model for human blood. J. Non-Newtonian Fluid Mech. 140, 57-70.

Pries, A. R., Neuhaus, D. \& Gaehtgens, P. 1992 Blood viscosity in tube flow: dependence on diameter and hematocrit. Am. J. Physiol. 263 (Heart Circ. Physiol. 32), H1770-H1778. 


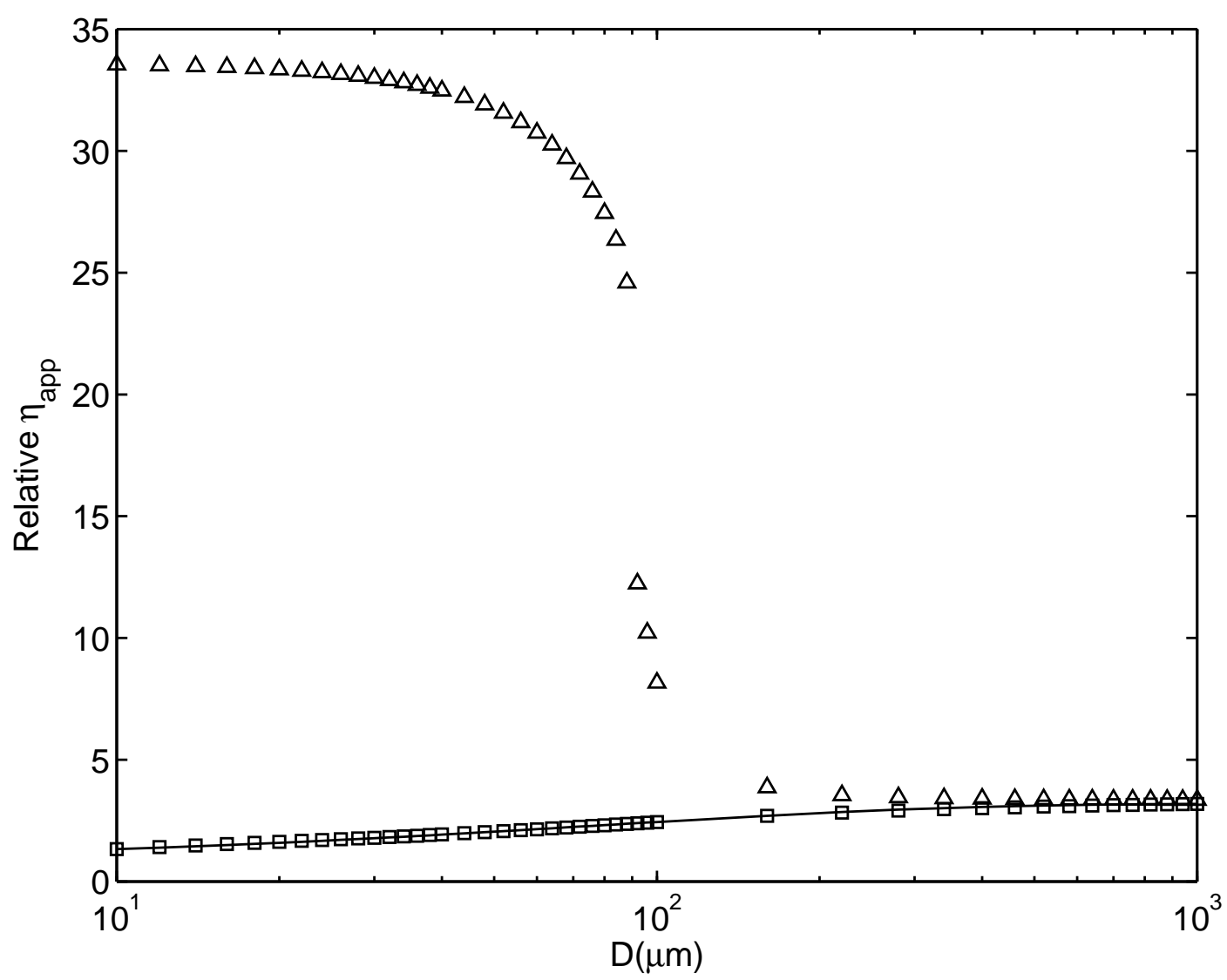

Figure 17. Fåhraeus-Lindqvist effect. Apparent relative viscosity $\eta_{a p p}$ against tube diameter $D$ $(\mu \mathrm{m}) . \quad P=-1000 \mathrm{Nm}^{-3} . \Delta$ : homogeneous model. $\square$ : non-homogeneous model. In both models $H_{c t} / H_{c}=H_{c}+\left(1-H_{c}\right)(1+1.7 \exp (-0.35 D)-0.6 \exp (-0.01 D))($ Pries et al. $(1990))$.

Pries, A. R. \& Secomb, T. W. 2005 Microvascular blood viscosity in vivo and the endothelial surface layer. Am. J. Physiol. Heart Circ. Physiol. 289, H2657-H2664.

Pries, A. R., Secomb, T. W., Gaentgens, P. \& Gross, J. F. 1990 Blood flow in microvascular networks. Experiments and simulation. Circ. Res. 67, 826-834.

Rossi, L. F., McKinley, G. \& Cook, L. P. 2006 Slippage and migration in Taylor-Couette flow of a model for dilute wormlike micellar solutions. J. Non-Newtonian Fluid Mech. 136, 79-92.

Secomb, T. W. 2003 Mechanics of red blood cells and blood flow in narrow tubes. In Modeling and Simulation of Capsules and Biological cells (ed. C. Pozrikidis), pp. 163-196. Boca Raton, Fl.: Chapman and Hall.

Sharan, M. \& Popel, A. S. 2001 A two-phase model for flow of blood in narrow tubes with increased effective viscosity near the wall. Biorheology 38, 415-428.

Shiga, T., Imaizumi, K., Harada, N. \& Sekiya, M. 1983 Kinetics of rouleaux formation using TV image analyzer. I. Human erythrocytes. Am. J. Physiol. 245, H252-H258. 
Sun, C. \& Munn, L. L. 2005 Particulate nature of blood determines macroscopic rheology: a 2D lattice Boltzmann analysis. Biophys. J. 88, 1635-1645.

Sutera, S. P., Seshadri, V., Croce, P. A. \& Hochmuth, R. M. 1970 Capillary blood flow : II. Deformable model cells in tube flow. Microvasc. Res. 2, 420-433.

Thurston, G. B. 1975 Elastic effects in pulsatile blood flow. Microvasc. Res. 9, 145-157.

Yen, R. T. \& Fung, Y. C. 1977 Inversion of Fahraeus effect and effect of mainstream flow on capillary hematocrit. Appl. Physiol.: Respirat. Environ. Exercise Physiol. 42, 578-586. 